CERN-SL-2000-069 (AP)

\title{
Tutorial on Linear Colliders
}

\author{
Frank Zimmermann \\ CERN, SL Division
}

\begin{abstract}
Proceeding from the collision point towards the source, we discuss purpose and design concepts of the various linear-collider subsystems, as well as important mechanisms of emittance dilution, beam diagnostics, and advanced tuning methods. In particular, we address beamstrahlung, linac emittance degradation due to dispersion and wake fields, scaling of damping-ring parameters with collider energy, fast beam-ion and electron-cloud instabilities, coherent synchrotron radiation, and rf guns. Five case studies are examined in detail.
\end{abstract}

Presented at Joint CERN-Japan-JINR-Russia-USA Accelerator School

JAS'2000, 1 to 14 July 2000

Geneva, Switzerland

October 6, 2000 


\section{Contents}

1 Perspective $\quad 2$

2 Beamstrahlung and Luminosity $\quad 5$

3 Final Focus $\quad 6$

4 Collimation $\quad 14$

5 Linac 16

5.1 Dispersion . . . . . . . . . . . . . . . . 16

5.2 Pulse-To-Pulse Stability . . . . . . . . . . . . . . . . . . 18

5.3 Wake Fields . . . . . . . . . . . . . . . . . . . . . 19

5.4 Acceleration . . . . . . . . . . . . . . . . . . 20

5.5 Quadrupole and Structure Misalignment . . . . . . . . . . . . . . . 25

5.6 Dispersion-Free Steering . . . . . . . . . . . . . . . . 26

5.7 Computer Simulations ． . . . . . . . . . . . . . . . . 30

6 Damping Rings $\quad 30$

6.1 Synchrotron Radiation . . . . . . . . . . . . . . . . . . . . . . . . . . . . . . . . . 30

6.2 Intrabeam Scattering . . . . . . . . . . . . . . . . . 32

6.3 Emittance Measurements . . . . . . . . . . . . . . . . . . . 33

6.4 Momentum Compaction Factor . . . . . . . . . . . . . . . . . . 36

6.5 Novel Instabilities . . . . . . . . . . . . . . . . . . . . . . 38

6.5 .1 Fast Beam-Ion Instability . . . . . . . . . . . . . . . . . . 38

6.5 .2 Electron-Cloud Instability . . . . . . . . . . . . . . . . . . . . 39

6.6 Coherent Synchrotron Radiation . . . . . . . . . . . . . . . . . . . 41

$7 \quad$ RF Gun and Positron Source $\quad 41$

\section{Perspective}

A linear electron-positron collider was first proposed by Tigner in 1965 [1] and later by Amaldi in 1975 [2]. Meanwhile, the Stanford Linear Collider (SLC) [3] has become a successful prototype. During the course of its operation from 1987 to 1998, the SLC performance was continually improved, as numerous advanced beam quality control techniques were invented and implemented $[4,5,6,7]$. We may take for granted that many of the techniques developed for the SLC will also be utilized at the next-generation linear colliders.

All future linear colliders will bring into collision highly energetic electron or positron beams, which are accelerated in two diametrically opposed linear accelerators (linacs). The first and only linear collider so far, the SLC, operated at a centre-of-mass energy of about $100 \mathrm{GeV}$ with rms interaction-point (IP) beam spot sizes of several hundred nanometers vertically and more than a micron horizontally. The next generation linear colliders aim for roughly 10 times higher energies and 100 times smaller vertical spot sizes. Table 1 compares IP beam parameters for the SLC with those proposed for various future projects. TESLA [8] coordinated by DESY is a superconducting (s.c.) linear collider, NLC [9] designed at SLAC uses a normal-conducting linac at 4 times the SLC rf frequency $(11.4 \mathrm{GHz}$ instead of $2.8 \mathrm{GHz}$ ), and CLIC $[10,11]$ studied at CERN operates at $30 \mathrm{GHz}$ 
and its power source is based on two-beam acceleration (see below). The differences between the projects reflect different design choices and emphases.

A linear accelerator (linac) consists of many successive arrays (structures) of coupled rf cavities (cells). In these cells a longitudinal rf electric field accelerates the electron or positron bunches to high energy. A larger accelerating field, or voltage gradient, is desirable, since it implies a shorter linac length for the same final beam energy. The accelerating gradient $G$ (in units of volts per meter) can be written as

$$
G=\sqrt{R P}
$$

with $P$ the supplied rf power and $R$ the so-called shunt impedance, both per unit length. The shunt impedance in turn may be expressed as $R=(R / Q) Q$, where $R / Q$ is a quantity that depends purely on the geometry of the accelerating cavity. Values of $R / Q$ equal to $200 \Omega$ per cavity length are common. $R / Q$ is reduced for larger iris radii, where the term 'iris' refers to the opening hole between successive cavity cells, through which both the beam and the rf wave propagate. The quality factor $Q$ is roughly equal to $V /\left(S \delta\left(\omega_{\mathrm{rf}}\right)\right)$ with $V$ the volume of the cavity, $S$ its surface area, and $\delta\left(\omega_{\mathrm{rf}}\right)$ the skin depth at the rf frequency [12]. For cavities in a superconducting linac, such as TESLA, the $Q$ value can be extremely high, even larger than $10^{11}$ [13]. That means it is possible to increase the iris apertures, so as to reduce the beam-induced 'wake' fields and, unavoidably, in parallel also the value of $(R / Q)$, but still retain a large shunt impedance $R$ and a modest rf power. On the other hand, the $Q$ value of normal-conducting cavities is much smaller, typically several times $10^{3}$ at $30 \mathrm{GHz}$ [14], and therefore it is important to preserve a large $R / Q$, even if this implies enhanced wake-field effects.

The higher frequencies chosen for the normal-conducting accelerating structures are based on the assumption that the achievable accelerating gradient increases roughly in proportion to the $\mathrm{rf}$ frequency, as would be the case if the field gradient is limited by rf breakdown or trapping of dark current generated by field emission $[15,16,17]$.

In conventional linacs, the rf energy is produced in devices called klystrons, which are powered by other devices called modulators. A klystron uses the bunching of a low-energy electron beam in response to a weak input rf signal to amplify the rf energy. The modulators among other components contain an energy storage unit, a fast switch, and a pulse-forming network. Usually the rf pulse generated by the klystrons is a factor 6-10 longer and weaker than required, and, therefore, it must be compressed. This is done by properly combining either parts of an rf pulse generated at different times from the same klystron or pulses from adjacent klystrons. The various rf compression schemes are known by their acronyms, such as SLED ('SLAC energy doubler'), SLED-II, or DLDS (delay line distribution system). In some of the proposed colliders, several thousands of klystrons and modulators are necessary to reach $1 \mathrm{TeV}$ centre-of-mass energy.

The number of klystrons and modulators is reduced drastically in the two-beam approach studied for CLIC. Here a low-energy intense 'drive beam' is employed to transport and compress the rf power which is initially produced in a separate low-frequency drive-beam linac. An attractive feature of CLIC is that there are no active rf components in the main linac, and that the high rf frequency chosen $(30 \mathrm{GHz})$ may allow reaching multiple $\mathrm{TeV}$ energies with a linac length comparable to that of the lower-energy lower-frequency designs. The overall layout of a 3-TeV CLIC is shown in Fig. 1. The figure also illustrates the generation of the drive beam and its distribution along the main linac.

The remainder of this report is structured as follows. In the next section, we discuss the constraints arising from the beam-beam interaction and the resulting luminosity scaling for linear colliders. We then describe design concepts, beam dynamics, operation, measurement challenges, and tuning methods for the various subsystems, proceeding against the beam direction from interaction 
Table 1: Beam and interaction-point parameters for various proposed linear colliders compared with those of the SLC. Note that some numbers may have changed since publication, and that, e.g., TESLA now contemplates an alternative parameter set with higher luminosity. [ ${ }^{\dagger}$ The SLC spot sizes quoted refer to the 1998 average values.]

\begin{tabular}{|lccccc|}
\hline parameter & symbol & SLC & TESLA & NLC & CLIC \\
\hline \hline c.m. energy [TeV] & $\mathrm{E}$ & 0.1 & 0.5 & 1 & 3 \\
luminosity [10 $\left.{ }^{34} \mathrm{~cm}^{-2} \mathrm{~s}^{-1}\right]$ & $L$ & 0.0002 & 0.84 & 1.3 & 10 \\
repetition rate [Hz] & $f_{\text {rep }}$ & 120 & 4 & 120 & 100 \\
bunch charge [10 $\left.{ }^{10}\right]$ & $N_{b}$ & 3.7 & 1.8 & 1.0 & 0.4 \\
bunches/rf pulse & $n_{b}$ & 1 & 2260 & 95 & 154 \\
bunch separation [ns] & $\Delta_{b}$ & - & 354 & $2.8 / 1.4$ & 0.67 \\
av. beam power [MW] & $P_{b}$ & 0.04 & 13 & 9 & 14.8 \\
bunch length [mm] & $\sigma_{z}$ & 1 & 0.5 & 0.12 & 0.03 \\
\hline hor. emittance $[\mu \mathrm{m}]$ & $\gamma \epsilon_{x}$ & 50 & 12 & 4.5 & 0.68 \\
vert. emittance $[\mu \mathrm{m}]$ & $\gamma \epsilon_{y}$ & 8 & 0.03 & 0.1 & 0.02 \\
hor. beta [mm] & $\beta_{x}^{*}$ & 2.8 & 25 & 12 & 8 \\
vert. beta [mm] & $\beta_{y}^{*}$ & 1.5 & 0.5 & 0.15 & 0.15 \\
hor. spot size [nm] & $\sigma_{x}^{*}$ & $1700^{\dagger}$ & 783 & 235 & 43 \\
vert. spot size [nm] & $\sigma_{y}^{*}$ & $900^{\dagger}$ & 5.5 & 4 & 1.0 \\
\hline Upsilon & $\Upsilon$ & $2 \times 10^{-3}$ & 0.02 & 0.3 & 8.1 \\
pinch enhancement & $H_{D}$ & 2.0 & 1.6 & 1.45 & 2.24 \\
beamstrahlung & $\delta_{B}[\%]$ & 0.06 & 1.0 & 10 & 31 \\
photons per $\mathrm{e}^{-}\left(\mathrm{e}^{+}\right)$ & $N_{\gamma}$ & 1 & 0.9 & 1.4 & 2.3 \\
\hline
\end{tabular}

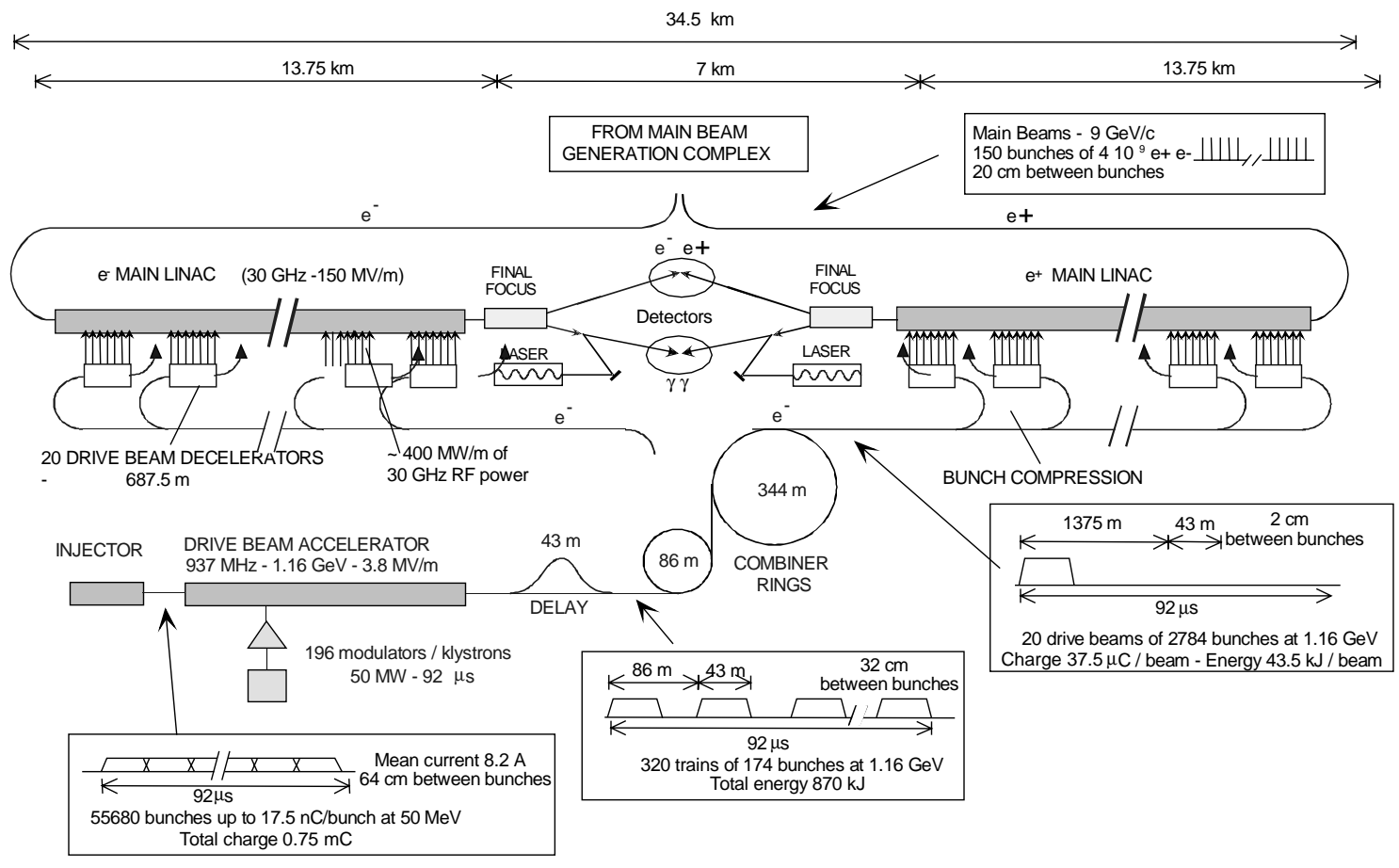

Figure 1: Schematic layout of the CLIC 3-TeV linear collider [10, 11]. 
point and final focus over collimation and linac towards damping rings and rf gun. Five illustrative case studies are presented in detail.

\section{Beamstrahlung and Luminosity}

Assuming Gaussian bunch distributions and ignoring the variation of the beam sizes during the collision, e.g., due to hourglass effect (depth of focus) or due to beam-beam forces ('pinch enhancement'), the luminosity of a linear collider can be written as

$$
L=\frac{f_{\mathrm{rep}} n_{b} N_{b}^{2}}{4 \pi \sigma_{x}^{*} \sigma_{y}^{*}}
$$

where $f_{\text {rep }}$ denotes the repetition rate at which beam and $\mathrm{rf}$ are sent through the linac, $n_{b}$ the number of bunches per rf pulse, $N_{b}$ the number of particles per bunch, $\sigma_{x}^{*}$ the rms horizontal beam size, and $\sigma_{y}^{*}$ the rms vertical beam size at the interaction point.

During the collision, individual electrons or positrons emit synchrotron radiation in the strong field of the opposing beam. This radiation is called beamstrahlung. To preserve a decent energy spectrum of the luminosity, the number of beamstrahlung photons emitted per electron, $N_{\gamma}$, must be limited to a value of the order of one. Considering this constraint and assuming flat beams with $\sigma_{x}^{*} \gg \sigma_{y}^{*}$ the luminosity formula above can be re-expressed as [18]

$$
L \approx\left(\frac{5}{r_{e}}\right) \frac{P_{\mathrm{wall}}}{E_{\mathrm{b}}} N_{\gamma} \frac{\eta}{\sigma_{y}^{*}}
$$

where $r_{e}$ is the classical electron radius, $P_{\text {wall }}$ the wall-plug power, $E_{\mathrm{b}}$ the final beam energy, and $\eta$ the conversion efficiency of wall-plug power into average beam power $\left(P_{\text {beam }}=f_{\text {rep }} E_{\mathrm{b}} N_{b} n_{b}\right)$. The beam energy is fixed by the physics requirements and the wall plug power limited by economical reasons. Hence, there are only two free parameters which can be optimized for maximum luminosity: the conversion efficiency $\eta$ and the vertical spot size $\sigma_{y}^{*}$.

At the SLC the parameter $\eta$ was much smaller than $1 \%$. For all future projects it is raised to roughly $10 \%$, for example, by increasing the number of bunches per rf pulse from 1 to about 100, and by improving the efficiency of all $\mathrm{rf}$ components. Clearly, the optimization of $\eta$ is being pushed to its limits.

The vertical spot size is the second free parameter. In all proposed designs, it is more than 100 times smaller than at the SLC. Such tiny spot sizes are achieved both by much reduced emittances and by interaction-point beta functions that are squeezed down to $150 \mu \mathrm{m}$, as is illustrated in Table 1 .

Many beam-dynamics challenges for the linear collider are related to the small spot size, for example, the design of the final-focus optics, stability tolerances, emittance preservation, and production of the low-emittance beam.

Let us now take a closer look at the beamstrahlung. The typical energy of the beamstrahlung photons is characterized by the parameter $\Upsilon$. This is equal to two thirds the classical critical energy divided by the beam energy $E_{\mathrm{b}}[19]$,

$$
\Upsilon=\frac{2}{3} \frac{\hbar \omega_{c}}{E_{\mathrm{b}}} \approx \frac{5}{6} \frac{\gamma r_{e}^{2} N}{\alpha \sigma_{z}\left(\sigma_{x}+\sigma_{y}\right)},
$$

where $\alpha$ denotes the fine structure constant. For synchrotron radiation emitted from a dipole magnet the critical freqency is $\omega_{c}=\frac{3}{2} c \gamma^{3} / \rho$ [20] and, usually, the energy of synchrotron radiation photons is much smaller than the beam energy $\hbar \omega_{c} \ll E_{\mathrm{b}}$. For the beamstrahlung emitted during the beambeam collision, this need not be the case. Typical values of $\Upsilon$ at the interaction point are $2 \times 10^{-3}$ 
for the SLC, 0.3 for the NLC, and almost 10 for CLIC. If $\Upsilon$ becomes comparable to 1 or larger, a significant portion of beamstrahlung photons convert into real electron-positron pairs in the strong electro-magnetic fields of the two beams. Unfortunately, linear colliders at multi-TeV energies can hardly avoid operating in this regime.

Besides $\Upsilon$, there is a second parameter of interest, namely the number of beamstrahlung photons emitted per electron. It reads [19]

$$
N_{\gamma} \approx \frac{5}{2} \frac{\alpha \sigma_{z}}{\gamma \lambda_{e}} \Upsilon\left[\frac{1}{\left(1+\Upsilon^{2 / 3}\right)^{1 / 2}}\right] \approx 2 \frac{\alpha r_{e} N_{b}}{\sigma_{x}+\sigma_{y}}
$$

The last approximation applies if $\Upsilon$ is small. For example, choosing $N=10^{10}$ and $N_{\gamma}=1$, we obtain $\left(\sigma_{x}+\sigma_{y}\right) \approx 400 \mathrm{~nm}$, consistent with the NLC parameter set.

Note that by reducing the bunch length $\sigma_{z}$, one can reach a parameter regime where $\Upsilon$ is large and the spot size small, but where we can still ensure that $N_{\gamma} \leq 1$, thanks to the quantum correction term - the square brackets - of Eq. (5). This is sometimes referred to as the quantum suppression of beamstrahlung. It arises, roughly speaking, from the fact that the electrons cannot radiate photons of energy higher than the beam energy. The classical spectrum of synchrotron-radiation photon energies is modified in this extreme quantum regime [19].

Two further quantities characterizing the beamstrahlung are the average energy loss per electron [19],

$$
\delta_{B} \approx \frac{1}{2} N_{\gamma} \Upsilon\left[\frac{\left(1+\Upsilon^{2 / 3}\right)^{1 / 2}}{\left(1+(1.5 \Upsilon)^{2 / 3}\right)^{2}}\right],
$$

and the fraction of luminosity at the nominal energy [21],

$$
\frac{\Delta L}{L} \approx \frac{1}{N_{\gamma}^{2}}\left(1-e^{-N_{\gamma}}\right)^{2},
$$

which depends only on $N_{\gamma}$. The value of $\Delta L / L$ drops rapidly for increasing $N_{\gamma}, e . g$, for $N_{\gamma}=1$, it is $81 \%$, for $N_{\gamma}=2$ only $25 \%$, and for $N_{\gamma}=3$ a bare $11 \%$.

Introducing the aspect ratio $r \equiv \sigma_{y} / \sigma_{x}$, the number of beamstrahlung photons scales as $N_{\gamma} \propto$ $N_{b} /\left(\sigma_{x}(1+r)\right)$ and the luminosity as $L \propto N_{b} N_{\gamma}(1+r) / r$. Hence, in order to maximize the luminosity while at the same time constraining the number of beamstrahlung photons, it is best to operate with flat beams where $r \ll 1$.

Flat-beam parameters have been adopted for all future linear collider designs. Various other methods to overcome the beamstrahlung problem were proposed in the past, such as 4-beam collisions [22, 23] (2 electron beams colliding with 2 positron beams, so that the net electric and magnetic fields are zero), plasma [24] (where the plasma return current cancels the beam fields), and photon-photon collisions $[25,26]$ (here the beam energy is converted into photon energy by Compton scattering off a high-power laser).

\section{Final Focus}

The small beta functions at the collision point are achieved by focusing the beam with strong quadrupole magnets located a few meters upstream. The normalized focusing strength of a quadrupole, $K$ (in units of $\mathrm{m}^{-2}$ ), depends on the particle momentum as

$$
K=\frac{B_{T}}{a(B \rho)}=\frac{e B_{T}}{a p_{0}(1+\delta)}
$$


where $B_{T}$ is the pole tip field, $a$ the pole-tip radius, $B \rho \equiv p / e$ the magnetic rigidity of the particle, $p_{0}$ the design momentum, and $\delta \equiv\left(p-p_{0}\right) / p_{0}$ the relative momentum deviation. Typical values for the final quadrupole are $B_{T} / a \approx 300-500 \mathrm{~T} / \mathrm{m}$ and $K \approx 0.1-0.3 \mathrm{~m}^{-2}$. Since the focusing strength is energy dependent, particles with different momentum deviations will be focused at different distances behind the quadrupole. The change in focal length with particle energy is called the chromaticity of the final focus. It can be computed as an integral over the final quadrupoles $\xi \approx \int d s \beta(s) K(s)$. If the chromaticity is not corrected the vertical spot size at the interaction point becomes

$$
\sigma_{y}^{*} \approx \sigma_{y, 0}^{*} \sqrt{1+\xi_{y}^{2} \delta_{\mathrm{rms}}^{2}}
$$

with $\sigma_{y, 0}^{*}\left(=\sqrt{\beta_{y}^{*} \epsilon_{y}}\right)$ the ideal linear design spot size and $\delta_{\text {rms }}$ the rms energy spread. Since typically $\xi \approx 30000$ and $\delta_{\text {rms }} \approx 0.28 \%$, if uncorrected, the chromaticity would increase the IP spot size by several orders of magnitude. Therefore, chromatic correction is indispensable. As in a storage ring, this correction is performed by placing sextupoles at locations with nonzero dispersion. Typically two pairs of sextupoles are used, for the horizontal and vertical chromatic correction, respectively. The sextupole pairs are placed a multiple of $\pi$ in betatron-phase advance away from the final quadrupoles. Figure 2 illustrates the basic layout of a final-focus system with chromatic correction.
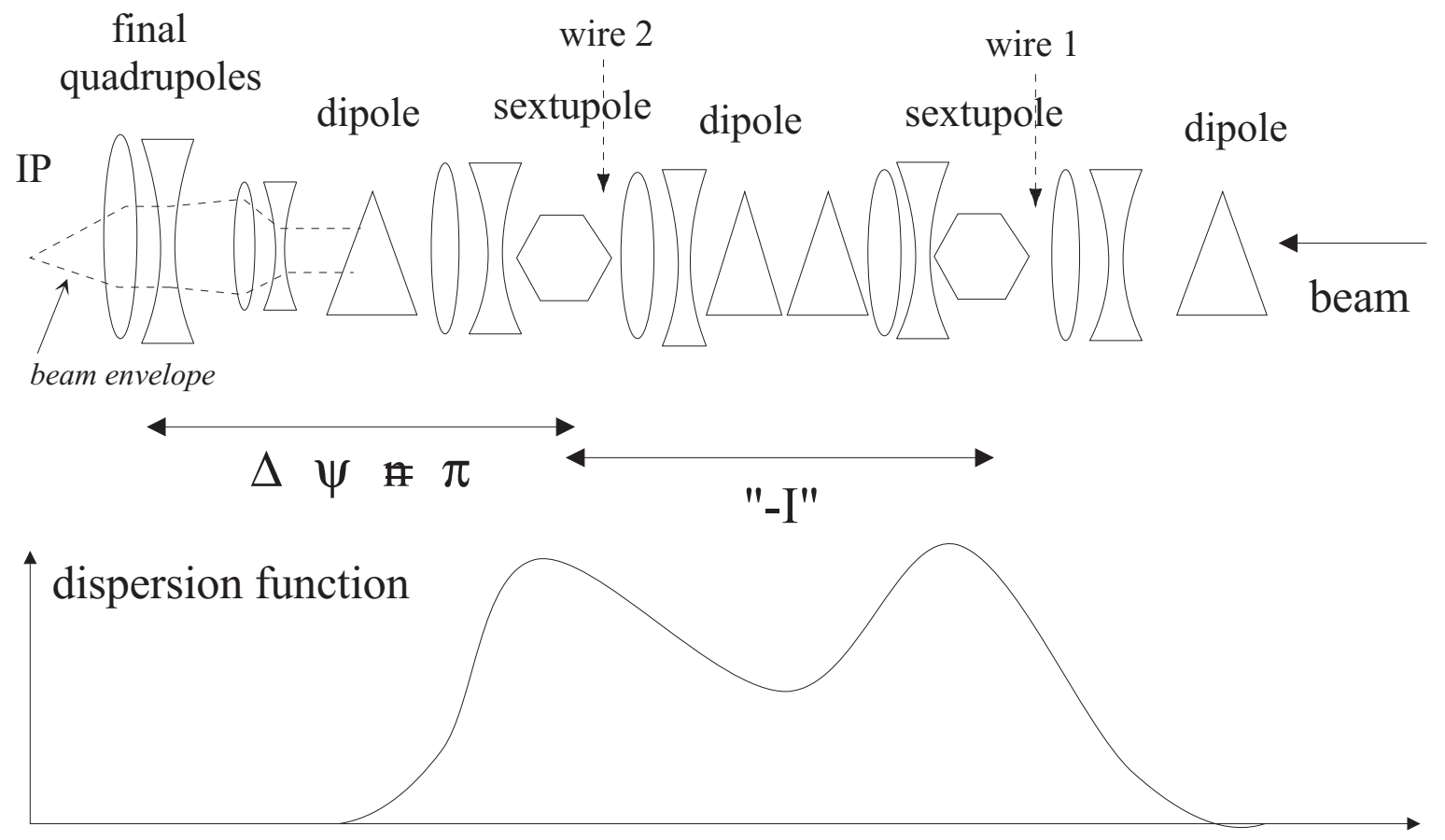

Figure 2: Schematic representation of a final-focus system. The beam moves from right to left. It passes the chromatic correction section and then a final demagnifier, before it finally reaches the interaction point, where it collides with an opposing beam. Only one pair of sextupoles is shown. A similar set of sextupoles and bending magnets would be located further on the right. Two pairs of sextupoles (4 sextupoles in total) are necessary in order to compensate the quadrupole chromaticity in both transverse planes and to globally cancel all unwanted low-order nonlinear aberrations induced by a single sextupole (quadratic kicks with amplitude and second-order dispersion) [27]. Wire scanners located near the two sextupoles, as indicated, can measure the energy-position correlation within the bunch; see case study I. 
The dispersion at the sextupoles is generated by bending magnets. Thus, we are faced with the undesirable situation that we need bending magnets at the highest energy of the linear collider. Although the bending magnets in the final focus are much weaker than those in a ring collider, the synchrotron radiation in these magnets is important and imposes severe design constraints. In particular, this synchrotron radiation is responsible for a dramatic increase in the length of conventional SLC-type final-focus systems, when extrapolated to TeV energies.

The linear beam transport in a single-pass system is often expressed by a $(6 \times 6)$ matrix relating the initial (subindex ' $i$ ') and final (subindex ' $f$ ') phase-space coordinates [28]:

$$
\left(\begin{array}{c}
x \\
x^{\prime} \\
y \\
y^{\prime} \\
z \\
\delta
\end{array}\right)_{f}=\left(\begin{array}{llllll}
R_{11} & R_{12} & R_{13} & R_{14} & R_{15} & R_{16} \\
R_{21} & R_{22} & R_{23} & R_{24} & R_{25} & R_{26} \\
R_{31} & R_{32} & R_{33} & R_{34} & R_{35} & R_{36} \\
R_{41} & R_{42} & R_{43} & R_{44} & R_{45} & R_{46} \\
R_{51} & R_{52} & R_{53} & R_{54} & R_{55} & R_{56} \\
R_{61} & R_{62} & R_{63} & R_{64} & R_{65} & R_{66}
\end{array}\right)\left(\begin{array}{c}
x \\
x^{\prime} \\
y \\
y^{\prime} \\
z \\
\delta
\end{array}\right)_{i}
$$

In general we can express the position of a particle or of the beam centroid as the sum of betatron oscillation, $x_{\beta}$, dispersion $D$ and higher order dispersion, $D^{(k)}(k=2, \ldots)$, as

$$
x=x_{\beta}+D \delta+\sum_{k \geq 2} D^{(k)} \delta^{k} .
$$

Often the higher-order dispersion terms $\left(D^{(k)}\right.$ with $\left.k \geq 2\right)$ are ignored, as we will do in the following analysis.

As already mentioned, in a conventional final focus, two pairs of sextupoles are used for chromatic correction, one pair for the horizontal plane, and the other for the vertical plane. We now consider one such pair in more detail. We assume that at the location of the first sextupole the dispersion is finite, $D_{i} \neq 0$, but that the slope of dispersion iz zero, $D_{i}^{\prime}=0$, and that there are no vertical bending magnets, hence $R_{36}=0$ and $R_{46}=0$. The $(2 \times 2)$ submatrices in the vertical and horizontal plane are commonly chosen as $-I$ (minus identity) transformations [27]. The full $R$ matrix between the two sextupoles forming a pair then has the form

$$
\left(\begin{array}{cccccc}
-1 & 0 & 0 & 0 & 0 & R_{16} \\
0 & -1 & 0 & 0 & 0 & R_{26} \\
0 & 0 & -1 & 0 & 0 & 0 \\
0 & 0 & 0 & -1 & 0 & 0 \\
0 & 0 & 0 & 0 & 1 & R_{56} \\
0 & 0 & 0 & 0 & 0 & 1
\end{array}\right)
$$

We write the initial position of a particle on a dispersive trajectory as $x_{i}=x_{\beta, i}+D_{i} \delta$, and the corresponding final position as $x_{f}=x_{\beta, f}+D_{f} \delta$. From Eqs. (10) and (12) it follows that $x_{\beta, f}=-x_{\beta, i}$ and that the dispersion at the second sextupole is equal to

$$
D_{f}=-D_{i}+R_{16}
$$

This illustrates that without bending magnets (i.e., with $R_{16}=0$ ) the dispersion propagates exactly like a betatron oscillation. If there are bending magnets between the initial and final positions, in general the $(1,6)$ matrix element is not zero, i.e., $R_{16} \neq 0$, and, in particular, the strengths of the bending magnets between the sextupoles can be adjusted so that $R_{16}=2 D_{i}$, whence $D_{f}=D_{i}$. In addition, it is possible to design the optics such that $R_{26}=0$, and hence $D_{i}^{\prime}=D_{f}^{\prime}=0$. The last 
nonzero element, $R_{56}$, describes the change in path length for different momentum deviations. It is the analogue of the momentum compaction factor in a storage ring. Usually, $R_{56}$ in the final focus is so small, that its effect can be ignored.

What exactly is the idea behind the $-I$ transform? We try to illustrate its merits. For simplicity we consider the horizontal plane only. We assume that the sextupoles are thin, so that their effect may be represented by a single nonlinear deflection, and we denote their integrated strengths by $K_{s, 1}$ and $K_{s, 2}$. The integrated strength of a sextupole in units of $\mathrm{m}^{-2}$ is defined as

$$
K_{s}=\left.l_{s} \frac{1}{B \rho} \frac{\partial^{2}}{\partial x^{2}} B(x)\right|_{x=0}=l_{s} \frac{B_{T}}{(B \rho) a^{2}},
$$

where $l_{s}$ denotes the length of the sextupole, $a$ its inner radius, $B_{T}$ the pole tip field at the radius $a$, and $B \rho(=p / e)$ the magnetic rigidity. As before, the dispersion at the sextupoles is taken to be equal to $D_{i} \neq 0$ and the slope to be zero $D_{i}^{\prime}=0$. We denote the particle coordinate just prior to the first sextupole by $x_{i}$, the associated trajectory slope by $x_{i}^{\prime}$, and the relative momentum deviation by $\delta$. Behind the first sextupole the slope of the particle trajectory becomes

$$
x_{1}^{\prime}=x_{i}^{\prime}+\frac{1}{2} K_{s, 1} x_{i}^{2}=x_{0}^{\prime}+\frac{1}{2} K_{s, 1} x_{\beta, i}^{2}+K_{s, 1} x_{\beta} D_{i} \delta+\frac{1}{2} K_{s, 1} D_{i}^{2} \delta^{2} .
$$

In addition to the initial slope, we here recognize three nonlinear dependencies introduced by the thin sextupole. The term proportional to $x_{\beta}^{2}$ represents a geometric aberration and the component quadratic in $\delta$ a second order dispersive term. The mixed product proportional to $x_{\beta} \delta$ is the chromatic term, which we want to generate in order to compensate the chromaticity of the final-focus quadrupoles.

Now applying the $-I$ transform, Eq. (12), with $R_{26}=0$ and $R_{16}=2 D_{i}$, we obtain the particle coordinates and slopes just prior to the second sextupole:

$$
\begin{gathered}
x_{2}=-x_{\beta}-D_{i} \delta+R_{16} \delta=-x_{\beta}+D_{i} \delta \\
x_{2}^{\prime}=-x_{\beta}^{\prime}-\frac{1}{2} K_{s, 1} x_{i}^{2} .
\end{gathered}
$$

Inserting $x_{i}=x_{\beta, i}+D_{i} \delta$ and applying the kick from the second quadrupole, $\Delta x^{\prime}=K_{s, 2} x_{2}^{2}$, we obtain

$$
x_{2}^{\prime}=-x_{\beta}^{\prime}-\frac{1}{2} K_{s, 1}\left(x_{\beta}^{2}+2 x_{\beta} D_{i} \delta+D_{i}^{2} \delta^{2}\right)+\frac{1}{2} K_{s, 2}\left(x_{\beta}^{2}-2 x_{\beta} D_{i} \delta+D_{i}^{2} \delta^{2}\right)
$$

For equal sextupole strengths, $K_{s, 1}=K_{s, 2} \equiv K_{s}$, the geometric aberrations and the second-order dispersion terms cancel exactly, and all that is left is the chromatic component:

$$
x_{2}^{\prime}=-x_{\beta}^{\prime}-2 K_{s} x_{\beta} D_{i} \delta .
$$

The important conclusion is that a $-I$ pair of sextupoles, as considered here, only generates chromaticity but no other low-order aberrations [27]. This conclusion still holds true if the vertical motion is also included in the analysis. Of course, in reality the $-I$ transform is not perfect, but itself varies with the momentum deviation. This gives rise to higher-order chromo-geometric aberrations, which ultimately limit the energy bandwidth of the final-focus system.

In circular accelerators dispersion is usually measured by sampling off-energy orbits with beamposition monitors. In linear colliders, however, varying the energy at some point in the beam line and observing the induced change in orbit measures the $R_{16}$ matrix element between the point of energy change and the BPMs downstream. In general this is not equal to the energy-position correlation within the bunch [29]. The energy-position correlation in the bunch is a result of changes 
in the individual particle energies, - due to acceleration, synchrotron radiation or wake fields, - and subsequent energy-dependent path lengths all along the beam line, whereas the $R_{16}$ measurement probes the effect of a change in acceleration at one particular location only.

\section{Case Study I: Beam Dispersion}

Conceive a scheme by which the horizontal and vertical energy-position correlation in the bunch can be monitored. Hint: one possibility is to use two sets of wire scanners, each with three wires tilted at different angles, e.g., at $90^{\circ}, 45^{\circ}$ and $135^{\circ}$ with respect to the horizontal plane, and separated by a -I optical transform with bending magnets as in Eq. (12). [29]. A wire scanner is a device which measures the horizontal or vertical or diagonal beam size. It is equipped with thin filaments of, e.g., $W$ or $C$, which are moved in small steps through the beam. Recording the scattering rate as a function of wire position and correcting for the finite size of the wire, one can determine the rms beam size at the wire location in the direction orthogonal to the wire filament.

Why could it be important to minimize such correlations in the beam?

Solution: We consider the -I transform of Eq. (12), and place two wire scanners at the initial and final locations. The $90^{\circ}$ (vertical) filament of the wire measures the horizontal spot size. The latter consists of a betatron part and a dispersive part, added in quadrature:

$$
\sigma_{x}=\sqrt{\beta_{x} \epsilon_{x}+\left(D_{x} \delta\right)^{2}} .
$$

We refer to the first wire by the subindex 1 and to the second wire by 2. The particle position at wire 1 is

$$
x_{1}=x_{\beta}+D_{x} \delta+\Delta D_{x} \delta .
$$

It transforms into

$$
x_{2}=-x_{\beta}+D_{x} \delta-\Delta D_{x} \delta
$$

at the second wire. The term $\Delta D_{x} \delta$ is the incoming beam dispersion mismatch (or, more precisely, the undesired energy-position correlation) which we want to measure and ultimately correct. This term propagates like a free betatron oscillation.

The rms horizontal beam sizes are computed by averaging over the beam distribution. The beam sizes at the two wire scanners are

$$
\begin{aligned}
& \sigma_{x 1}^{2}=\left\langle x_{1}\right\rangle^{2}=\left\langle x_{\beta}^{2}\right\rangle+\left(D_{x}+\Delta D_{x}\right)^{2}\left\langle\delta^{2}\right\rangle, \\
& \sigma_{x 2}^{2}=\left\langle x_{2}\right\rangle^{2}=\left\langle x_{\beta}^{2}\right\rangle+\left(D_{x}-\Delta D_{x}\right)^{2}\left\langle\delta^{2}\right\rangle,
\end{aligned}
$$

with $\left\langle x_{\beta}^{2}\right\rangle=\beta_{x} \epsilon_{x}$ and $\left\langle\delta^{2}\right\rangle=\sigma_{\delta}^{2}$. The angular brackets indicate an average over the beam distribution. Without horizontal dispersion mismatch $\left(\Delta D_{x}=0\right)$, the horizontal beam size measured on the two wires is identical. The difference in the squared beam sizes thus provides a measure of the mismatch,

$$
\sigma_{x 1}^{2}-\sigma_{x 2}^{2}=4 D_{x} \Delta D_{x} \sigma_{\delta}^{2}
$$

or [29]

$$
\Delta D_{x}=\frac{\sigma_{x 1}^{2}-\sigma_{x 2}^{2}}{4 D_{x} \sigma_{\delta}^{2}},
$$

where $D_{x}$ is the (matched) design dispersion at the wire, and the rms momentum spread $\sigma_{\delta}$ must be obtained from another measurement, typically from an additional wire scan at a location with large dispersion.

Next we look at the situation in the vertical plane. The vertical particle position at the first wire is

$$
y_{1}=y_{\beta}+\Delta D_{y} \delta,
$$


which, at the second wire, transforms into

$$
y_{2}=-y_{\beta}-\Delta D_{y} \delta
$$

where $\Delta D_{y}$ denotes the vertical dispersion mismatch. Due to the absence of vertical bending, the vertical beam sizes at the two wires are always equal, independent of the amount of vertical dispersion mismatch. The reason is that both terms on the right-hand side of Eq. (27) change sign, and that a constant term, such as $D_{x}$ horizontally, is missing. However, beam sizes measured on wire filaments tilted at $130^{\circ}$ and $35^{\circ}$ depend on the product of the vertical dispersion mismatch and the horizontal design dispersion, and, thereby, they allow us to determine the vertical dispersion. A wire scanner of this type is sketched in Fig. 3. The tilted wires measure the beam size in the $u$ and $v$

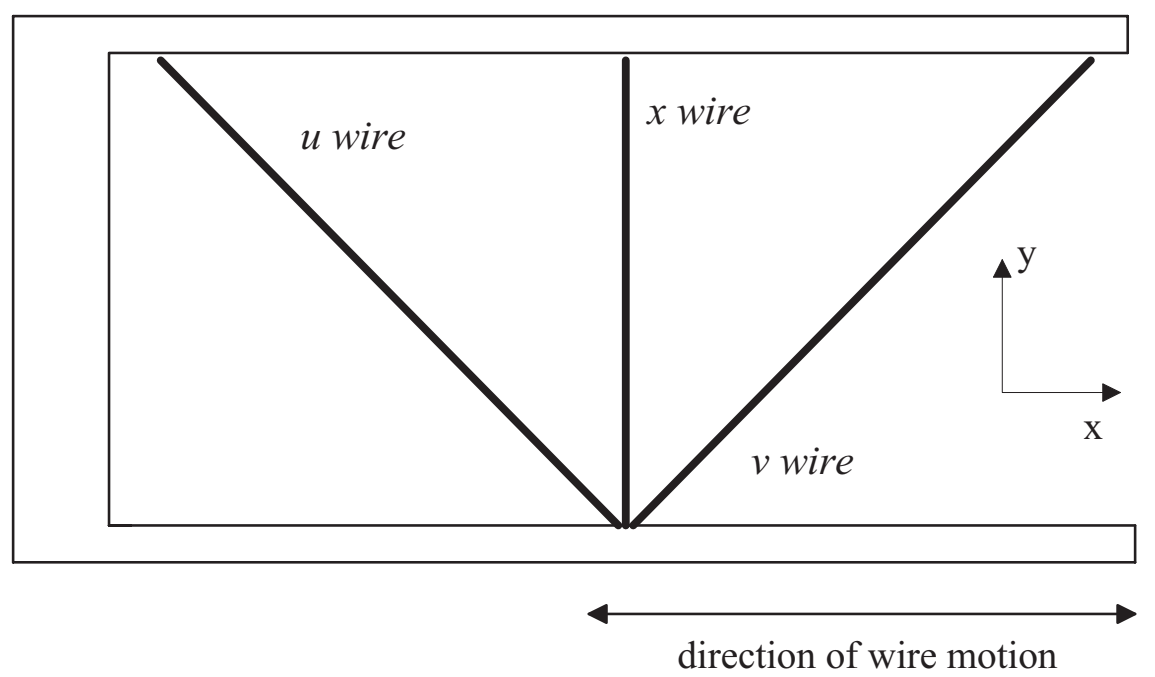

Figure 3: Horizontal wire-scanner mount equipped with 3 filaments, which measure the beam size in the $x, u$ and $v$ directions

directions defined by

$$
\begin{aligned}
& u=\frac{x+y}{\sqrt{2}}, \\
& v=\frac{x-y}{\sqrt{2}} .
\end{aligned}
$$

Computing the rms beam sizes at these tilted wires we find

$$
\begin{aligned}
\sigma_{u}^{2} & =\left\langle u^{2}\right\rangle=\frac{1}{2}\left(\sigma_{x}^{2}+\sigma_{y}^{2}\right)+\langle x y\rangle, \\
\sigma_{v}^{2} & =\left\langle v^{2}\right\rangle=\frac{1}{2}\left(\sigma_{x}^{2}+\sigma_{y}^{2}\right)-\langle x y\rangle,
\end{aligned}
$$

so that, for each wire scanner,

$$
\sigma_{u}^{2}-\sigma_{v}^{2}=2\langle x y\rangle
$$

Inserting the expressions for $x$ and $y$, Eqs. (21), (22), (27), and (28), the correlation $\langle x y\rangle$ can be written

$$
\begin{aligned}
& \left\langle x_{1} y_{1}\right\rangle=\left\langle x_{\beta} y_{\beta}\right\rangle+\Delta D_{y}\left(D_{x}+\Delta D_{x}\right) \sigma_{\delta}^{2} \\
& \left\langle x_{2} y_{2}\right\rangle=\left\langle x_{\beta} y_{\beta}\right\rangle+\Delta D_{y}\left(-D_{x}+\Delta D_{x}\right) \sigma_{\delta}^{2}
\end{aligned}
$$


Subtracting these two expressions, the terms $\left\langle x_{\beta} y_{\beta}\right\rangle$ (betatron coupling) and the terms proportional to the horizontal mismatch, $\Delta D_{x}$, cancel, and it remains

$$
\left\langle x_{1} y_{1}\right\rangle-\left\langle x_{2} y_{2}\right\rangle=2 D_{x} \Delta D_{y} \sigma_{\delta}^{2} .
$$

Re-expressing $\langle x y\rangle$ in terms of $\frac{1}{2}\left(\sigma_{u}^{2}-\sigma_{v}^{2}\right)$, and using Eq. (33), the vertical dispersion mismatch is found [29]:

$$
\Delta D_{y}=\frac{\sigma_{u 1}^{2}-\sigma_{v 1}^{2}-\sigma_{u 2}^{2}+\sigma_{v 2}^{2}}{4 D_{x} \sigma_{\delta}^{2}} .
$$

The dispersion in a transport line is not uniquely defined. The dispersion mismatch we have considered here is related to the energy-position correlation within the initial bunch distribution. This can me made explicit by writing, e.g., the vertical dispersion mismatch as

$$
\Delta D_{y}=\frac{\left\langle y_{1} \delta\right\rangle}{\left\langle\delta^{2}\right\rangle}
$$

Correction of the mismatch implies that we remove the correlated component, so as to obtain the new position coordinate

$$
y_{\text {cor }}=y_{1}-\Delta D_{y} \delta=y_{1}-\frac{\left\langle y_{1} \delta\right\rangle}{\left\langle\delta^{2}\right\rangle} \delta .
$$

Squaring and averaging over the distribution we find

$$
\left\langle y_{\text {cor }}^{2}\right\rangle=\left\langle y_{1}^{2}\right\rangle-\frac{\left\langle y_{1} \delta\right\rangle^{2}}{\left\langle\delta^{2}\right\rangle} \leq\left\langle y_{1}^{2}\right\rangle .
$$

Hence, the beam size after correction, $\left\langle y_{\mathrm{cor}}^{2}\right\rangle^{1 / 2}$, is always smaller than a beam size with some residual correlations, $\left\langle y_{1}^{2}\right\rangle^{1 / 2}$.

In our example, the wire scanners are located a betatron-phase advance of $90^{\circ}$ or an integer multiple thereof away from the interaction point (IP). In this case, minimizing the beam size at the wires amounts to minimizing the IP beam divergence.

There are many design constraints for a conventional final focus system:

1) The additional energy spread due to synchrotron radiation emitted in a bending magnet is

$$
\delta_{\mathrm{sr}}^{2} \approx \frac{55}{24 \sqrt{3}} r_{e} \lambda_{e} \gamma^{5} \frac{\theta^{3}}{l_{b}^{2}}
$$

with $\gamma=E /\left(m_{e} c^{2}\right), r_{e}$ the classical electron radius, $\theta$ the bending angle and $l_{b}$ the length of the dipole magnet. If the radiation occurs after the (first) sextupoles, this energy spread is not chromatically corrected and it increases the IP spot size as

$$
\frac{\Delta \sigma_{y}^{*}}{\sigma_{y 0}^{*}}=\xi_{y} \delta_{\mathrm{sr}} .
$$

The term in Eq. (42) must be added in quadrature to the design spot size $\sigma_{y 0}$, yielding a total spot size

$$
\sigma_{y}^{*}=\sqrt{\sigma_{y 0}^{* 2}+\Delta \sigma_{y}^{* 2}}
$$

2) Since the chromaticity is proportional to the beta function at the final quadrupoles, it grows roughly inversely with the IP beta function,

$$
\xi_{y} \approx l^{*} / \beta_{y}^{*}
$$


where $l^{*}$ denotes the effective free length from the last quadrupole to the IP.

3) The condition for the chromatic correction of the final quadrupoles by upstream sextupoles with integrated strength $K_{s}$, in units of $\mathrm{m}^{-2}$, reads

$$
\left|2 D_{s} K_{s} \beta_{s}\right|=\xi_{y}
$$

where $D_{s}$ and $\beta_{s}$ are the horizontal dispersion and vertical beta function at the two sextupoles.

4) The dispersion scales with bending angle and length as

$$
D_{s} \propto l_{b} \theta
$$

5) A quadrupole gradient error $\Delta K$ in the final focus will shift the waist position longitudinally away from the collision point. This will cause a spot-size increase

$$
\frac{\Delta \sigma_{y}^{*}}{\sigma_{y}^{*}}=\Delta K \beta,
$$

where $\beta$ is the beta function at the location of the perturbation and we have assumed a phase advance to the IP equal to an odd multiple of $\pi / 2$, as is the case for most final-focus magnets. Again, $\Delta \sigma_{y}^{*}$ is added in quadrature; see Eq. (43).

In particular, changes in the horizontal orbit, $\Delta x$, at the second sextupole of a pair, e.g. due to vibration or position drifts of quadrupoles located between the two sextupoles, induce a quadrupole component, $\Delta K=K_{s} \Delta x$, and, thus, increase the IP beam spot size. The tolerance on the orbit stability at the second sextupole, with regard to orbit perturbations generated between the pair of sextupoles, is

$$
\Delta x<\frac{1}{5 K_{s} \beta_{s}},
$$

corresponding to a $2 \%$ increase in the absolute spot size.

\section{Case Study II: Length Scaling of Final-Focus Systems}

Assuming that the length of the final focus, $l_{\mathrm{FF}}$, increases in proportion to the length of the bending magnets, derive a scaling law for $l_{\mathrm{FF}}$ as a function of $\gamma, \beta^{*}$ and $\Delta x$.

Solution: Combining Eqs. (41) and (46), we find that

$$
\delta_{\mathrm{rms}} \propto \frac{D_{s}^{3 / 2}}{l_{\mathrm{FF}}^{5 / 2}} \gamma^{5 / 2} .
$$

Together with Eqs. (42) and (44), and limiting the blow up $\Delta \sigma_{y}^{*} / \sigma_{y}^{*}$ to, e.g., a value of 0.2, this translates into

$$
\frac{D_{s}^{3 / 2} \gamma^{5 / 2}}{l_{\mathrm{FF}}^{5 / 2}} \frac{l^{*}}{\beta_{y}^{*}}<\text { constant } .
$$

Solving Eqs. (48) and (45) for $D_{s}$ and using Eq. (44), one finds

$$
D_{s}>\frac{5}{2} \Delta x \xi_{y}=\frac{5}{2} \Delta x \frac{l^{*}}{\beta_{y}^{*}} .
$$

Inserting this into Eq. (50), we obtain the desired scaling law [30]

$$
l_{\mathrm{FF}} \propto \gamma \Delta x^{3 / 5} \frac{l^{*}}{\beta_{y}^{*}} .
$$


This scaling law predicts many kilometers or even tens of kilometers of final-focus lengths for a few-TeV collider. Therefore, novel final-focus concepts which may allow for a shorter system are presently under investigation. One approach is to perform the chromatic correction with sextupoles near the final quadrupole, accepting a nonzero slope of dispersion at the collision point [31]. Another approach is the use of a high-frequency rf quadrupole, which can compensate for the correlated energy spread across the bunch [32].

\section{Collimation}

Collimators are special elements which are positioned closest to the beam. Located somewhere between the linac and the interaction point, their primary function is to remove beam halo at large amplitudes, typically corresponding to 10-20 times the horizontal or 50-80 times the vertical rms beam size. Additional collimation may be required also before or in the linac.

The removal of the halo particles is necessary, since, if lost at aperture restrictions in the final focus or near the collision point, they can produce electromagnetic showers, muons and neutrons, or, if traversing the final quadrupoles with a large transverse offset, they can emit wide-angle synchrotron radiation. In either case, the halo particles may cause unacceptable background in the particle-physics detector. For an ideal linear collider there are only a few unavoidable sources of beam halo, such as scattering off residual gas or thermal photons (blackbody radiation). According to conservative estimates, the known scattering sources result in only about $10^{2}-10^{4}$ halo particles per bunch train $[33,34]$.

On the other hand, at the SLC, a large number of collimators were added over the first years of operation in an attempt to render the experimental conditions acceptable. Occasionally, more than $10 \%$ of the beam had to be scraped at the collimators over periods of hours. There was little quantitave understanding or modeling of the observed halo at the SLC, but the suspected culprits include magnet nonlinearities in the bunch compressor, longitudinal microwave instability in the damping rings, beam dynamics in the linac, and higher-order dispersion.

It is expected that at future colliders an improved design of damping rings and ring-to-linac transfer lines as well as a pre-collimation stage in front of the linac will reduce the halo reaching the end of the linac by several orders of magnitude compared with the SLC value.

The collimators do not only remove halo, but they also serve a second purpose. In case of a failure (e.g., mis-firing of ring extraction kicker or missing drive beam), they are the elements first hit by a mis-steered beam. Therefore, a common collimator design requirement is that the collimators should survive the single impact of one entire bunch train. After the loss of a mis-steered bunch train and prior to the next linac pulse, the accelerator can be switched off, and then restart with a smaller number of low-charge bunches.

For the parameters of all future linear colliders, guaranteeing the collimator survival is a major challenge. Already at the SLC, which should have operated in a safe regime, many collimators were damaged by the beam [35]. The collimator survival condition can be written as a lower limit on the beam size at the collimator [36]

$$
\sigma_{x} \sigma_{y}>\frac{\alpha_{T} Y}{\sigma_{\mathrm{UTS}} C_{p}} \frac{d \mathcal{E}}{d x}\left(\frac{n_{b} N_{b}}{2 \pi}\right)
$$

with $\sigma_{\mathrm{UTS}}$ the ultimate tensile strength, $\alpha_{T}$ the linear thermal expansion coefficient, $C_{p}$ the heat capacity, $Y$ the elastic modulus, $d \mathcal{E} / d x$ the specific energy loss per unit length, $n_{b}$ the number of bunches in the train, and $N_{b}$ the bunch population. 
As an example, taking material properties of copper, $\alpha=1.7 \times 10^{-5} \mathrm{~K}^{-1}, E=120 \mathrm{GPa}$, $C_{p}=0.385 \mathrm{Jg}^{-1} \mathrm{~K}^{-1}, d \mathcal{E} / d x \approx 1.44 \mathrm{MeV} \mathrm{cm} / \mathrm{g}$, and $\sigma_{U T S}=300 \mathrm{MPa}$, we find

$$
\left(\sigma_{x} \sigma_{y}\right)^{1 / 2} \geq 200 \mu \mathrm{m} \quad \text { or } \quad \beta_{x, y} \geq 1000 \mathrm{~km},
$$

where, in the last step, we have assumed normalized emittances of $\gamma \epsilon_{x}=0.68 \mu \mathrm{m}$ and $\gamma \epsilon_{y}=$ $0.02 \mu \mathrm{m}$ at $1.5-\mathrm{TeV}$ beam energy. Such enormous beta functions imply a long system and tight tolerances.

Fortunately, the above estimates are too pessimistic. For many conceivable failure modes, the emittance will blow up considerably before the beam hits the collimator. An example is shown in Fig. 4. In the simulation of a mis-steered beam, rapid filamentation due to large energy spread leads to an emittance increase by two or three order of magnitude. This suggests that the nominal beta functions at the collimators could be reduced accordingly. The required values then would appear more reasonable.

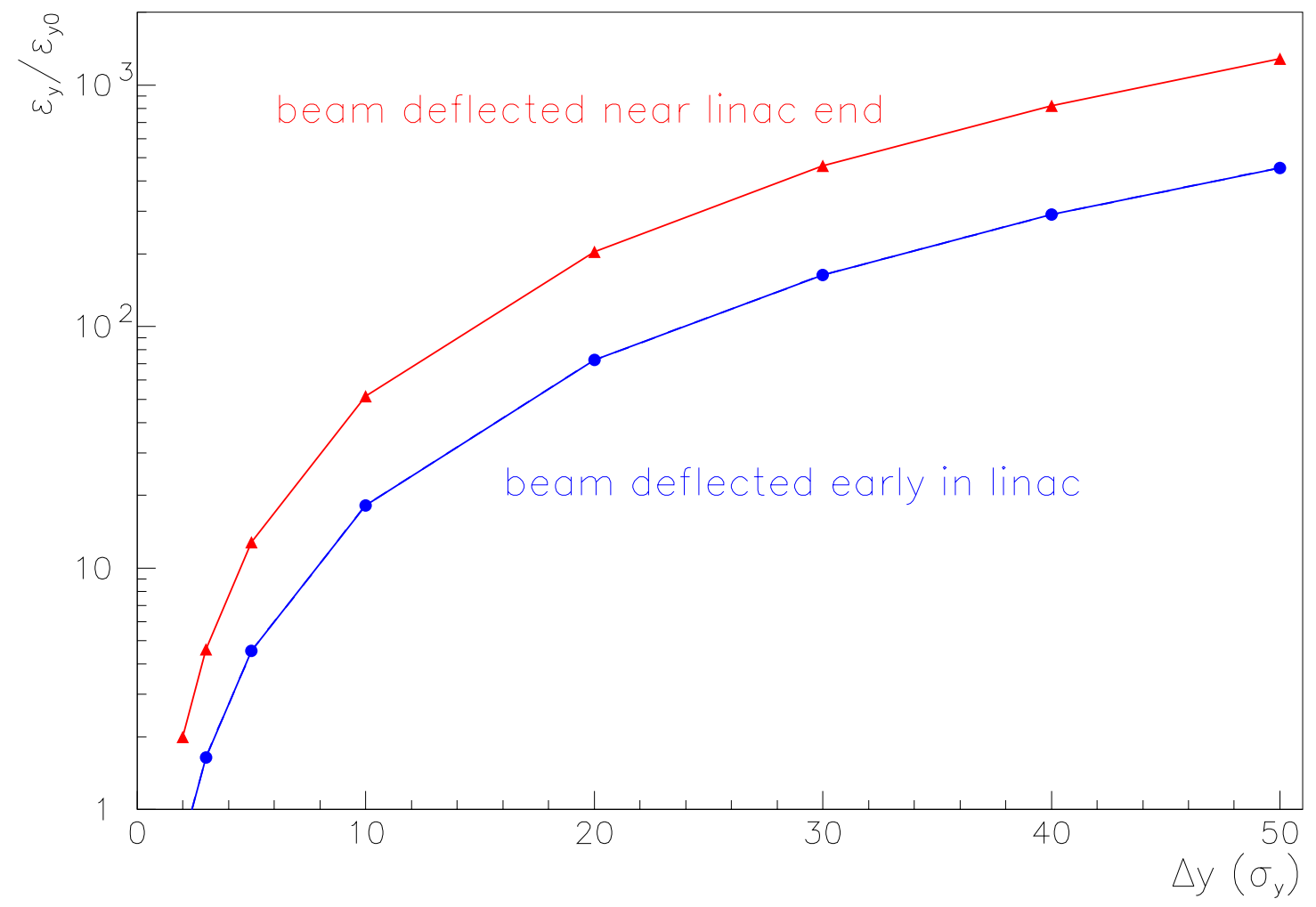

Figure 4: Emittance growth of a beam deflected in the early (bottom curve) or late part (top curve) of the linac as a function of induced oscillation amplitude in units of the rms beam size [37]. Because of rapid filamentation an unstable beam is accompanied by significant emittance blow up.

The development of a workable collimator system is still a matter of active research. The final design will depend on the answers to the following questions:

- How large is the beam halo?

- How many muons produced per bunch train are acceptable for the detector? 
- Which are the failure modes that can mis-steer the beam, and what is the resulting emittance increase for each of these?

The NLC design is considering the deployment of replacable or renewable collimators [38].

\section{Linac}

The linac is the heartpiece of a linear collider. It should not only accelerate the beam to high energy, but in addition preserve the transverse emittance and also supply the beam stably to the downstream final-focus and collimation systems. There are many perturbation sources which can cause pulse-topulse variation in the linac trajectory, for example, mechanical vibration of magnet supports, ground motion, small changes in the initial beam distribution from the damping ring, or drifts or fluctuations in the rf systems. Most dangerous are changes which occur from one rf pulse to the next, because it is extremely difficult to counteract these by feedback.

\subsection{Dispersion}

We will now look more closely at the beam dynamics in the linac, in order to understand how the emittance growth can occur. Both the beam centroid and the individual beam particles perform betatron oscillations along the linac. If we ignore the energy change due to acceleration, the betatron oscillation for arbitary momentum error $\delta$ is a solution of Hill's equation

$$
x^{\prime \prime}+\frac{K(s)}{1+\delta} x=0,
$$

where the quadrupole focusing strength $K(s)$ in units of $\mathrm{m}^{-2}$ is a function of the longitudinal position $s$, and the prime denotes the derivative with respect to $s$. In the presence of dipole magnets or quadrupole misalignments, the right hand-side of this equation would contain an inhomogeneous term $1 / \rho$, but we will ignore this for the moment. We next use a smooth approximation and replace the $s$ dependent force by a constant average. Instead of Eq. (54), we thus write

$$
x^{\prime \prime}+\frac{K}{1+\delta} x=0
$$

where we may identify $K=\langle K(s)\rangle_{s}$ with $1 / \beta^{2}$, the inverse square of the smoothed beta function. The solution can be written as an expansion in $\delta$ :

$$
x=x_{\beta}+D \delta+D^{(2)} \delta^{2}+\ldots
$$

where $D$ is called the dispersion and $D^{(2)}$ the second order dispersion. Inserting this solution into Eq. (55) and equating terms with equal powers of $\delta$, we obtain an equation for the on-energy betatron motion

$$
x_{\beta}^{\prime \prime}+K x_{\beta}=0
$$

and, from the terms of first order in $\delta$, an equation for the dispersion,

$$
D^{\prime \prime}+K D=K x_{\beta}
$$

According to Eqs. (57) and (58) a betatron oscillation propogating through the linac resonantly drives the dispersion: the natural oscillation frequencies of $x_{\beta}$ and $D$ are identical and $x_{\beta}$ enters as an excitation on the right-hand side of Eq. (58). 
Dispersion in a linac arises from deflections by misaligned quadrupoles or by wake fields excited by a beam passing off-centre through an accelerating structure. We can estimate the magnitude of these effects considering a two-particle model, where the bunch is represented by a leading and a trailing (macro-)particle, denoted by ' 1 ' and ' 2 ', each with half the bunch charge and separated by, e.g., twice the rms bunch length. For simplicity, we will ignore acceleration and use a smooth approximation for the betatron motion. We first look at the leading particle.

\section{Case Study III: Linac Dispersion}

(1) Dispersion for free betatron oscillation. Consider a beam deflected by an angle $\theta$ at $s=0$. The betatron motion of the leading particle is described by

$$
x_{1}^{\prime \prime}(s)+\frac{k_{\beta}^{2}}{1+\delta_{1}} x_{1}(s)=\frac{\theta}{1+\delta_{1}} \delta(s)
$$

where $\delta(s)$ is the Dirac delta function, indicating a single deflection of strength $\theta$ at location $s=0$, and $k_{\beta}=\sqrt{K}$ is the wave number of the betatron oscillation. Equation (59) describes the motion of an individual off-momentum particle in the head of the bunch, as well as the centroid motion of the bunch head if the latter experiences a centroid momentum offset $\delta_{1}$. The equality of the particle and centroid motion (for small bunch charges) is an advantage, since the single-particle dispersion can be measured by observing the response of the centroid motion to an energy error.

Solve Eq. (59), linearize the solution in $\delta_{1}$ and determine the dispersion for $k_{\beta} s \delta_{1} \ll 1$.

Solution: This is the equation of a harmonic oscillator. The solution reads [39]

$$
x_{1}(s)=\frac{\theta}{k_{\beta} \sqrt{1+\delta_{1}}} \sin \frac{k_{\beta} s}{\sqrt{1+\delta_{1}}} \approx \frac{\theta}{k_{\beta} \sqrt{1+\delta_{1}}}\left[\sin k_{\beta} s-\frac{1}{2} k_{\beta} s \delta_{1} \cos k_{\beta} s\right]
$$

or

$$
x_{1}(s) \approx \frac{\theta}{k_{\beta}} \sin k_{\beta} s-\frac{1}{2}\left[\theta s \cos k_{\beta} s+\frac{\theta}{k_{\beta}} \sin k_{\beta} s\right] \delta_{1}+\mathcal{O}\left(\delta_{1}^{2}\right) .
$$

From the term linear in $\delta_{1}$ we infer the dispersion at the bunch head,

$$
D_{1}(s)=-\frac{1}{2}\left[\theta s \cos k_{\beta} s+\frac{\theta}{k_{\beta}} \sin k_{\beta} s\right] .
$$

The solution is illustrated in Fig. 5. The linear increase with s reflects that the dispersion is resonantly driven [39].

(2) Dispersion behind $\pi$ bump. Orbit correction can be thought of as a superposition of $\pi$ bumps. Calculate the dispersion generated by a bump, represented by two kicks $\theta$, at $s_{1}=0$ and $s_{2}=\pi / k_{\beta}$.

Solution: The dispersion generated by a single kick at $s=0, D_{1}(s)$, was computed in Eq. (62). The dispersion generated by the second kick is obtained by simply shifting the argument by $s_{2}$, i.e., it is given by $D_{1}\left(s-\pi / k_{\beta}\right)$. The dispersion arising from the $\pi$ bump is then the sum of the terms generated by the two kicks [39]:

$$
D_{\pi}=D_{1}(s)+D_{1}\left(s-\pi / k_{\beta}\right)=-\frac{\theta \pi}{2 k_{\beta}} \cos k_{\beta} s .
$$

The solution is illustrated in Fig. 6. While the orbit after the $\pi$ bump is zero, the dispersion propagates at a constant amplitude. A perfectly centred orbit in the downstream linac section does not imply that the dispersion is zero as well.

The linearly growing dispersion component in a linac arises from the energy dependence of the oscillation frequency (chromaticity). Note that the definition of the linac dispersion $D_{1}$ differs from the periodic dispersion in a storage ring. Nevertheless, also the latter can be efficiently controlled by 'resonant' orbit bumps [40]. 


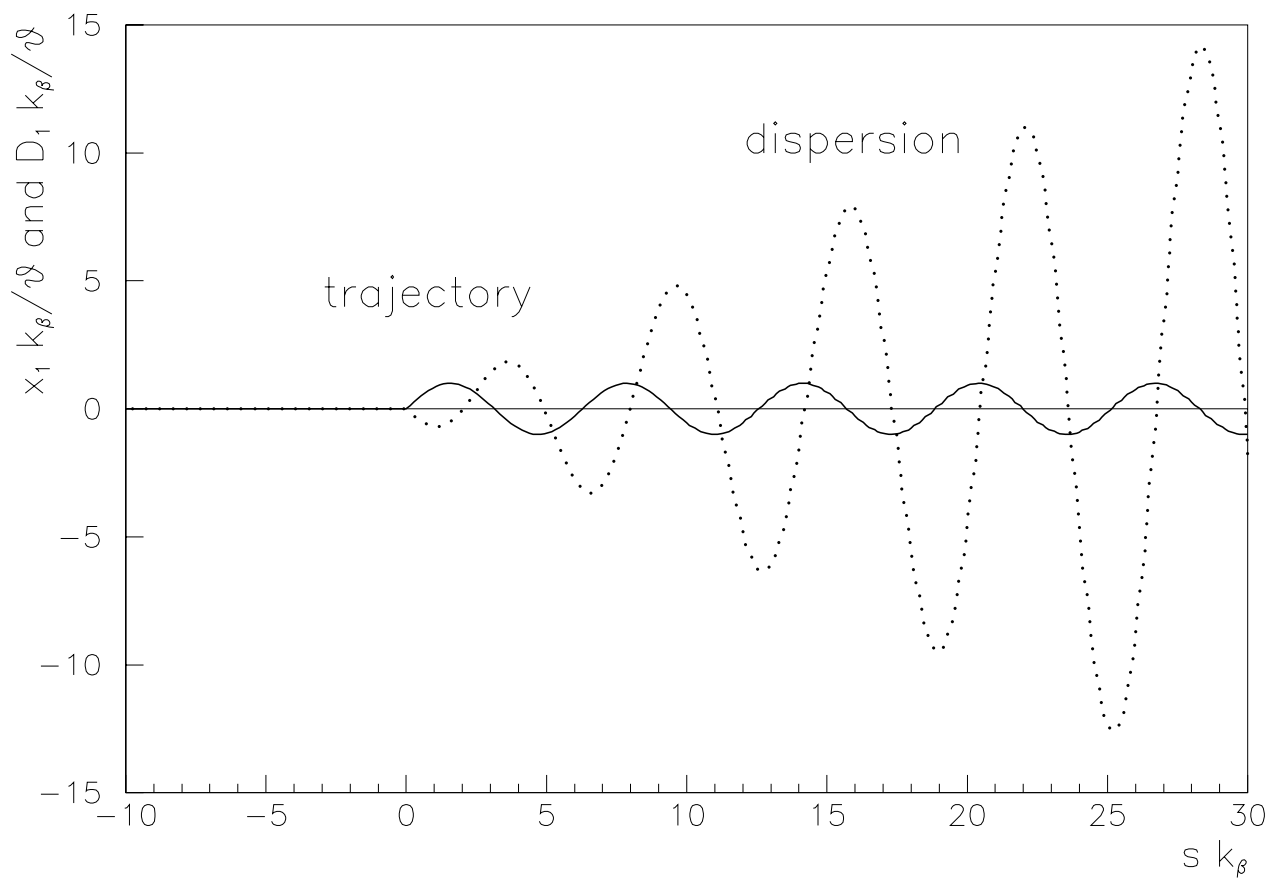

Figure 5: Trajectory oscillation, $x_{1} k_{\beta} / \theta$ for $\delta_{1}=0$, and resonantly growing dispersion at the bunch head, $D_{1} k_{\beta} / \theta$, induced by a deflection at $s=0$, according to Eqs. (61) and (62).

\subsection{Pulse-To-Pulse Stability}

Consider the situation where the vertical positions of the linac quadrupoles vary from one pulse to the next. Each of the misaligned quadrupoles will induce a betatron oscillation travelling down the linac. These betatron oscillations in turn give rise to dispersion. Due to the resonant excitation the dispersion grows much faster than the betatron motion. For this reason, the blow up of the final beam size at the end of the linac due to energy spread and accumulated dispersion may cause a larger luminosity loss than the beam-beam separation induced by the centroid betatron motion.

If the $i$ th quadrupole at location $i$ is misaligned by an amount $\Delta y$, the beam is deflected by $\theta_{i}=$ $K_{q}^{i} \Delta y$, where $K_{q}$ denotes the integrated strength of the quadrupole (in units of $\mathrm{m}^{-1}$ ). From the above solution, the amplitude of the resulting dispersion at the end of the linac is $D_{i}=\frac{1}{2} \theta_{i}\left(L-s_{i}\right)$, with $L$ denoting the full linac length and $s_{i}$ the position of the quadrupole. If a total of $N_{q}$ quadrupoles randomly shift in position by an rms value $\Delta y_{\text {rms }}$ the expectation value for the final dispersion is the incoherent sum of the individual contributions, and a rough estimate for the rms dispersion at the end of the linac reads [43]

$$
D_{\mathrm{rms}}=\frac{1}{4 \sqrt{2}} \theta_{\mathrm{rms}} L \sqrt{N_{q}}=\frac{1}{4 \sqrt{2}} K_{q} L \sqrt{N_{q}} \Delta y_{\mathrm{rms}} .
$$

The factor $1 / \sqrt{2}$ is the rms value of the cosine function in Eq. (62), where we have assumed a uniform distribution of phase advances between the quadrupoles and the IP. In order to re-express $K_{q}$ in terms of the average beta function, we note that the betatron phase advance over a linac FODO cell of length $L_{\text {cell }}$ is $\phi_{\text {cell }} \approx L_{\text {cell }} / \beta \approx \pi / 2$. The relation is strictly true for a $90^{\circ}$ lattice. In addition, the cell length and the quadrupole strength are roughly related as $L_{\text {cell }} \approx 2 \sqrt{2} / K_{q}$, and we thus 


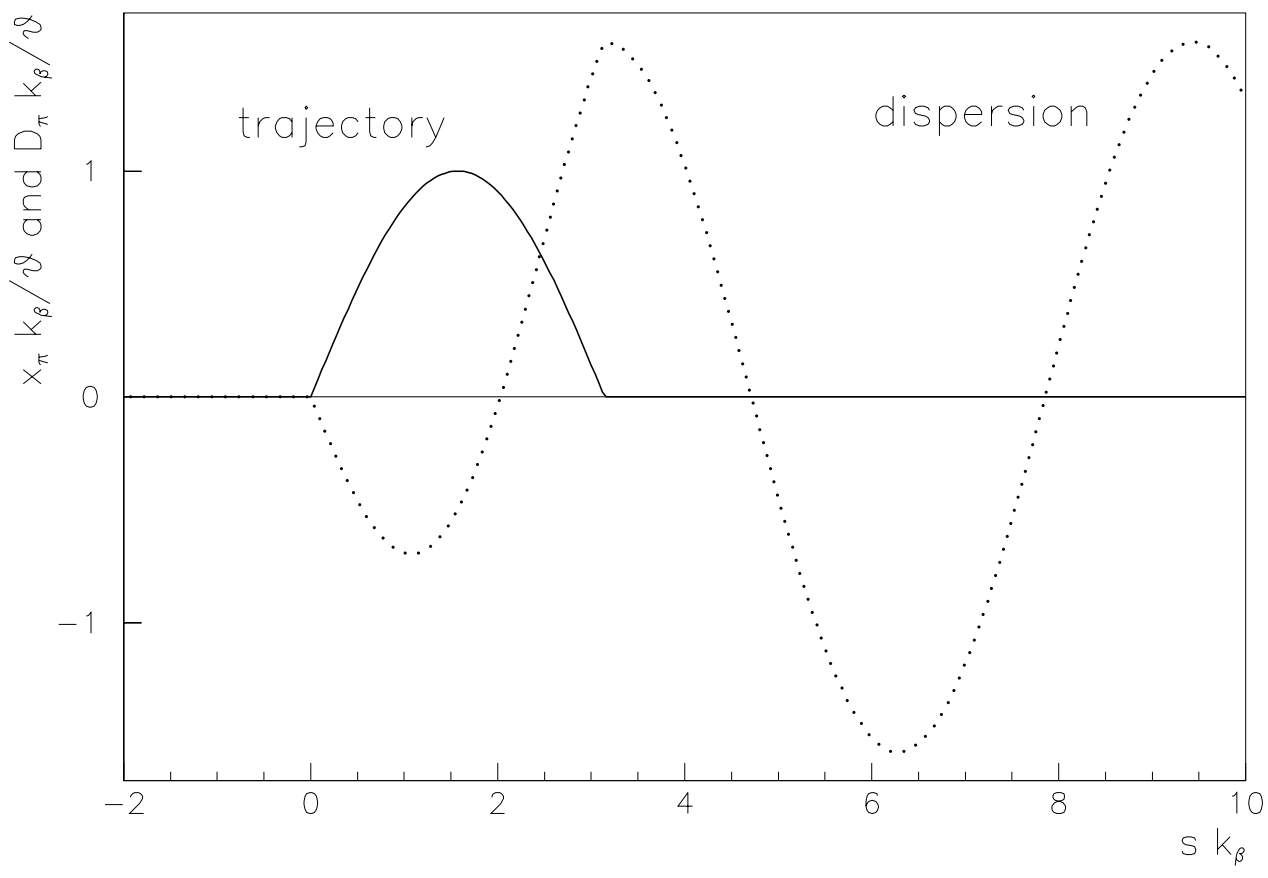

Figure 6: Trajectory perturbation, $x_{\pi} k_{\beta} / \theta$, and subsequent constant dispersion, $D_{\pi} k_{\beta} / \theta$, induced by a $\pi$ bump, according to Eq. (63).

approximate $K_{q} \approx 2 / \beta$. Note that again we have considered a constant beam energy and a smooth beta function.

Typically we require that the absolute beam size increase due to the additional dispersion generated between two linac pulses,

$$
\Delta \sigma_{y}=\left(\sqrt{\sigma_{y}^{2}+\left(D_{\mathrm{rms}} \delta_{\mathrm{rms}}\right)^{2}}-\sigma_{y}\right),
$$

should be less than $2 \%$, or $D_{\text {rms }} \delta_{\text {rms }}<\sigma_{y} / 5$, where $\sigma_{y}=\sqrt{\epsilon_{y} \beta_{y}}$ is the rms beam size. We then obtain the following tolerance on the pulse-to-pulse quadrupole stability:

$$
\Delta y_{\mathrm{rms}}<\frac{2 \sqrt{2} \sigma_{y} \beta}{5 \sqrt{N_{q}} \delta_{\mathrm{rms}} L} .
$$

Inserting parameters that approximately correspond to the CLIC linac, $\beta_{y} \approx 7 \mathrm{~m}, \gamma \epsilon_{y} \approx 5 \times 10^{-9}$ $\mathrm{m}, L=15 \mathrm{~km}, \delta_{\mathrm{rms}} \approx 1 \%, \gamma \approx 2 \times 10^{4}$, and $N_{q}=1500$, we find a tight tolerance: $\delta y_{\mathrm{rms}} \leq 1$ $\mathrm{nm}$, i.e., between two pulses the rms quadrupole motion in the CLIC linac should be less than 1 nanometer! The example parameters were chosen such that this estimate agrees with the result of an elaborate simulation [41], which includes the actual energy profile along the bunch, wake fields and acceleration. Refined analytical formulae and many other details on emittance preservation for linear colliders can be found in Ref. [42].

\subsection{Wake Fields}

Until now we have only looked at dispersion. Dispersion increases the emittance because of the nonzero energy spread across the bunch. A second important effect which degrades the linac emit- 
tance are transverse wake fields. The effect of the single-bunch wake field can be illustrated using the two-particle model [12]. For simplicity, we consider only the case without momentum deviation $\left(\delta_{1}=\delta_{2}=0\right)$. We have seen already that the bunch head performs the usual betatron motion

$$
x_{1}^{\prime \prime}+k_{\beta}^{2} x_{1}=0
$$

and that the solution of Eq. (67) is $x_{1}=\hat{x} \cos k_{\beta} s$. In the two-particle model, the equation of motion for the trailing macro-particle reads

$$
x_{2}^{\prime \prime}+k_{\beta}^{2} x_{2}=-\frac{N_{b} r_{e} W_{1}}{2 \gamma} x_{1} .
$$

The term on the right-hand side represents the wake field excited by the leading particle with a charge of $N_{b} / 2$. The strength of the wake, $W_{1}$, in units of inverse cubic length, depends on the distance of the bunch head and tail, hence on the rms bunch length. Typically, for short bunches, $W_{1}<0$ and the wake is 'defocusing', i.e., the tune shift for a rigid coherent betatron oscillation is negative.

Since the oscillation frequencies of $x_{1}$ and $x_{2}$ are the same, the trailing particle is resonantly driven. The solution of Eq. (68) becomes

$$
x_{2}=\hat{x} \cos k_{\beta} s-\Upsilon_{\mathrm{BBU}} \frac{s}{L} \hat{x} \sin k_{\beta} s
$$

where

$$
\Upsilon_{\mathrm{BBU}}=-\frac{N_{b} r_{e} W_{1} L}{4 \gamma \sqrt{K}}
$$

is called the beam break up parameter, and $L$ denotes the total length of the linac. We have chosen the initial conditions $x_{1}(0)=x_{2}(0)$ and $x_{1}^{\prime}(0)=x_{2}^{\prime}(0)=0$.

\subsection{Acceleration}

So far we have completely ignored the main task of a linac, which is to accelerate, i.e., to increase the beam energy. If we include acceleration, Eq. (67) for the head particle is replaced by

$$
\frac{d}{d s}\left[\gamma(s) \frac{d x_{1}}{d s}\right]+k_{\beta}^{2} x_{1}=0
$$

where $\gamma(s)=\gamma_{i}(1+\alpha s)$ is the relativistic factor, with $\gamma_{i}=\gamma(0)$ and $\alpha$ is a constant proportional to the acceleration gradient. We here again assume $\delta_{1}=\delta_{2}=0$. The solution to Eq. (71) depends on the way the betatron wavenumber varies along the linac. Most future designs assume a scaling close to

$$
k_{\beta} \approx \text { constant }
$$

which implies

$$
\beta \approx \gamma^{1 / 2}
$$

This scaling can be realized by increasing the lengths of all elements as $\sqrt{\gamma}$ and keeping the quadrupole pole-tip fields constant. Introducing the new independent variable $z=\sqrt{1+\alpha s}$, Eq. (71) is rewritten as

$$
z^{2} \frac{d^{2}}{d z^{2}} x_{1}+z \frac{d}{d z} x_{1}+4 \frac{k_{\beta}^{2}}{\alpha^{2}} z^{2} x_{1}=0
$$


This is a Bessel equation. For the initial condition $x_{1}(0)=\hat{x}, x_{1}^{\prime}(0)=0$ the solution for the head of the bunch reads

$$
\begin{aligned}
x_{1}= & -\frac{\pi k_{\beta}}{\alpha} \hat{x}\left[Y_{1}\left(\frac{2 k_{\beta}}{\alpha}\right) J_{0}\left(\frac{2 k_{\beta}}{\alpha} \sqrt{1+\alpha s}\right)\right. \\
& \left.-Y_{0}\left(\frac{2 k_{\beta}}{\alpha}\right) J_{1}\left(\frac{2 k_{\beta}}{\alpha} \sqrt{1+\alpha s}\right)\right] .
\end{aligned}
$$

A useful relation exists between the modified Bessel functions $Y_{0,1}$ and $J_{0,1}$,

$$
Y_{0}(w) J_{1}(w)-Y_{1}(w) J_{0}(w)=\frac{2}{\pi w}
$$

and, for large arguments, $w \gg 1$, these Bessel functions may be further approximated as

$$
\begin{aligned}
& J_{0}(w) \approx-Y_{1}(w) \approx \sqrt{\frac{2}{\pi w}} \cos \left(w-\frac{\pi}{4}\right), \\
& J_{1}(w) \approx Y_{0}(w) \approx \sqrt{\frac{2}{\pi w}} \sin \left(w-\frac{\pi}{4}\right) .
\end{aligned}
$$

Using the above relations, we can rewrite our solution as

$$
x_{1}(s) \approx \hat{x}\left[\frac{1}{(1+\alpha s)^{1 / 4}} \cos \left(\frac{2 k_{\beta}}{\alpha}(1-\sqrt{1+\alpha s})\right)\right] .
$$

Note that the oscillation amplitude decreases as $\left|x_{1}\right| \propto \sqrt{\beta / \gamma} \sim \gamma^{-1 / 4}$, due to the combined effect of adiabatic damping and variation in focusing strength along the linac.

The equation of motion for the tail particles again includes the wake field:

$$
x_{2}^{\prime \prime}+\frac{d \gamma / d s}{\gamma} x_{2}^{\prime}+k_{\beta}^{2} x_{2}=-\frac{N r_{0} W_{1}}{2 \gamma} x_{1}
$$

with $z=\sqrt{1+\alpha s}$. The solution is

$$
x_{2}(z)=x_{1}(z)-\frac{2 N r_{0} W_{1}}{\gamma \alpha^{2}} \int_{1}^{z} d z^{\prime} G\left(z, z^{\prime}\right) y_{1}\left(z^{\prime}\right)
$$

with the Green function

$$
G\left(z, z^{\prime}\right)=\frac{\pi}{2} z^{\prime}\left\{J_{0}\left[\frac{2 k_{\beta}}{\alpha} z^{\prime}\right] Y_{0}\left[\frac{2 k_{\beta}}{\alpha} z\right]-Y_{0}\left[\frac{2 k_{\beta}}{\alpha} z^{\prime}\right] J_{0}\left[\frac{2 k_{\beta}}{\alpha} z\right]\right\} .
$$

In the limit $\alpha \ll k_{\beta}$ this simplifies to

$$
G\left(z, z^{\prime}\right) \approx \sqrt{\frac{z^{\prime}}{z}} \frac{\alpha}{2 k_{\beta}} \sin \left(\frac{2 k_{\beta}}{\alpha}\left(z-z^{\prime}\right)\right)
$$

where again $z=\sqrt{1+\alpha s}$. The final solution is

$$
\begin{aligned}
x_{2}(s) \approx & \hat{x} \frac{1}{(1+\alpha s)^{1 / 4}}\left[\cos \left(\frac{2 k_{\beta}}{\alpha}(\sqrt{1+\alpha s}-1)\right)\right. \\
& \left.-\frac{N r_{0} W_{1}\left(\sigma_{z}\right)}{2 \gamma_{i} \alpha k_{\beta}}(\sqrt{1+\alpha s}-1) \sin \left(\frac{2 k_{\beta}}{\alpha}(\sqrt{1+\alpha s}-1)\right)\right] .
\end{aligned}
$$


Hence, for $s=L$ and $\alpha L \gg 1$, the beam break up parameter with acceleration is

$$
\Upsilon_{\mathrm{BBU}}=-\frac{N W_{1} r_{0} L}{2 k_{\beta}} \frac{1}{\sqrt{\gamma_{f} \gamma_{i}}}
$$

It can be obtained from the no-acceleration result by simply replacing $1 / \gamma$ with $2 / \sqrt{\gamma_{f} \gamma_{i}}$.

Assuming a constant beta function along the linac instead of $\beta \propto \gamma^{1 / 2}$, a similar expression is found; see Ref. [12]. In this case, the factor $1 / \gamma$ in the no-acceleration version of $\Upsilon_{\mathrm{BBU}}$ must be replaced by $1 / \gamma_{f} \ln \left(\gamma_{f} / \gamma_{i}\right)$.

Thus, it is often convenient to perform calculations first without acceleration and then to include the latter at the end using the appropriate substitution rule.

For the Stanford Linear Collider (SLC), $\Upsilon_{\mathrm{BBU}} \approx 15$, which means that if the beam was injected with a certain betatron oscillation, the oscillation of the tail increased by a factor of 15 along the linac. For the proposed 3-TeV CLIC linac, we compute $\Upsilon_{\mathrm{BBU}} \approx 5$.

(3) Dispersion with wake field. The equation of motion for the tail of the bunch includes the effect of the wake generated by the bunch head as an additional excitation term on the right-hand side,

$$
x_{2}^{\prime \prime}(s)+\frac{k_{\beta}^{2}}{1+\delta_{2}} x_{2}(s)=\frac{\theta}{1+\delta_{2}} \delta(s)-\frac{N r_{0} W_{1}}{2 \gamma\left(1+\delta_{2}\right)} x_{1}(s) .
$$

Solve this equation for $\delta_{1}=0$, determine the trajectory for $\delta_{2}=0$, and, by linearizing in $\delta_{2}$, the additional dispersion arising from the wake field, possibly using the beam break up parameter $\Upsilon_{\mathrm{BBU}}$. Acceleration can be approximately included 'a posteriori' by inserting the correctly modified value of $\Upsilon_{\mathrm{BBU}}$. The motion of the head particle was calculated above. For $\delta_{1}=0$ it reads

$$
x_{1}(s)=\frac{\theta}{k_{\beta}} \sin k_{\beta} s
$$

Solution: The solution is the sum of an oscillation at the natural frequency of the tail particle, $k_{\beta} / \sqrt{1+\delta_{2}}$ and a response to the head driving force at frequency $k_{\beta}$. We thus make the ansatz

$$
x_{2}(s)=A \sin k_{\beta} s+B \sin \frac{k_{\beta}}{1+\delta_{2}}+C \cos \frac{k_{\beta}}{1+\delta_{2}} s .
$$

Inserting this ansatz into Eq. (84) and also considering the two initial conditions $x_{2}(0)=0$ and $x_{2}^{\prime}(0)=x_{1}^{\prime}(0)=\theta$, we can solve for the three constants of integration, $A, B$ and $C$. We then obtain the solution of Eq. (84) as [39]

$$
x_{2}(s)=\frac{\theta}{k_{\beta}}\left(\frac{1}{\sqrt{1+\delta_{2}}} \sin \frac{k_{\beta} s}{\sqrt{1+\delta_{2}}}-\frac{\Upsilon_{\mathrm{BBU}} 2}{L k_{\beta} \delta_{2}}\left(\sin k_{\beta} s-\sqrt{1+\delta_{2}} \sin \frac{k_{\beta} s}{\sqrt{1+\delta_{2}}}\right)\right) .
$$

For $k_{\beta} s \delta_{2} \ll 1$ we can again expand in $\delta_{2}$. We find

$$
\begin{aligned}
x_{2}(s)= & \frac{\theta}{k_{\beta}} \sin k_{\beta} s-\frac{\Upsilon_{\mathrm{BBU}} \theta}{k_{\beta} L}\left(s \cos k_{\beta} s-\frac{1}{k_{\beta}} \sin k_{\beta} s\right) \\
& -\frac{\Upsilon_{\mathrm{BBU}} \theta}{4 k_{\beta} L}\left(k_{\beta} s^{2} \sin k_{\beta} s-s \cos k_{\beta} s+\frac{1}{k_{\beta}} \sin k_{\beta} s\right) \delta_{2} \\
& -\frac{1}{2}\left(\theta s \cos k_{\beta} s+\frac{\theta}{k_{\beta}} \sin k_{\beta} s\right) \delta_{2} .
\end{aligned}
$$


We identify the term linear in $\delta_{2}$ with the tail dispersion

$$
\begin{aligned}
D_{2}(s)= & -\frac{\Upsilon_{\mathrm{BBU}} \theta}{4 k_{\beta} L}\left(k_{\beta} s^{2} \sin k_{\beta} s-s \cos k_{\beta} s+\frac{1}{k_{\beta}} \sin k_{\beta} s\right) \\
& -\frac{1}{2}\left(\theta s \cos k_{\beta} s+\frac{\theta}{k_{\beta}} \sin k_{\beta} s\right) .
\end{aligned}
$$

The solution is sketched in Fig. 7. Note that the wake-induced dispersion is doubly resonantly driven, and grows quadratically with distance!

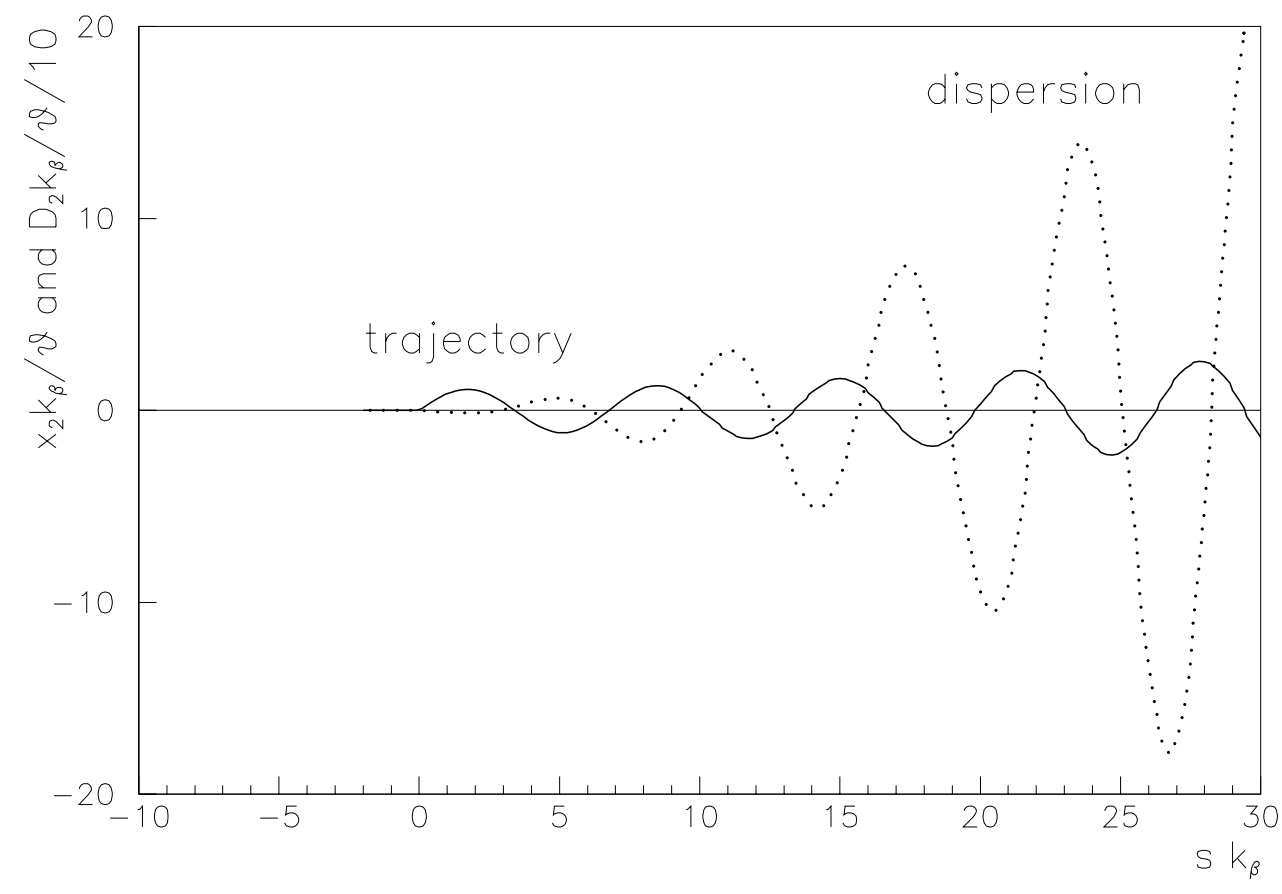

Figure 7: Growing oscillation of the trailing macroparticle, $x_{2} k_{\beta} / \theta$, due to beam break up, and the doubly resonantly driven tail dispersion, $D_{2} k_{\beta} / \theta / 10$, induced by a deflection at $s=0$ according to Eq. (87), for $\Upsilon_{\mathrm{BBU}} /\left(k_{\beta} L\right)=0.08$ (SLC value).

At the end of the linac the amplitude ratio of the wake-induced dispersion and the regular dispersion (without the wake-field effect) generated by a deflection early in the linac is $\Upsilon_{\mathrm{BBU}} / 2$. Hence, at the SLC, with $\Upsilon_{\mathrm{BBU}} \approx 15$, the wake-induced dispersion was about 7 times larger.

(4) BNS damping. The effect of the wake field on the tail of the bunch can be partially compensated by increasing the focusing strength for the tail particles, from $k_{\beta}$ to $k_{\beta}+\Delta k_{\beta}$ [44]. For a free betatron oscillation propagating through the linac, the additional focusing counteracts the wake-field 'defocusing' for a coherent betatron oscillation. To this end either rf quadrupoles with rapidly varying field can be used [45] or the position of the bunch can be adjusted with respect to the crest of the rf wave so that the tail acquires less energy than the head.

With BNS damping the equation of motion for the trailing macroparticle is

$$
x_{2}^{\prime \prime}(s)+\frac{\left(k_{\beta}^{2}+\Delta k_{\beta}^{2}\right)}{1+\delta_{1}} x_{2}(s)=\frac{\theta}{1+\delta_{2}} \delta(s)-\frac{N r_{0} W_{1}}{2 \gamma\left(1+\delta_{2}\right)} x_{1}(s)
$$


where again $x_{1}(s)=\frac{\theta}{k_{\beta}} \sin k_{\beta} s$ assuming $\delta_{1}=0$. BNS damping is achieved if

$$
\left(1+\frac{\Delta k_{\beta}}{k_{\beta}}\right)^{2}=1+\frac{2 \Upsilon_{\mathrm{BBU}}}{k_{\beta} L_{0}} .
$$

Show that under this condition and with $\delta_{2}=0$ the orbit of a trailing particle is identical to that of the bunch head: $x_{2}(s)=x_{1}(s)$.

Evaluate the dispersion of the trailing particle $x_{1}$ with BNS damping, assuming $\Delta k_{\beta} \ll k_{\beta}$, $\delta \ll 1, k_{\beta} s \delta \ll 1, \Upsilon_{\mathrm{BBU}} /\left(k_{\beta} L\right) \ll 1$, and $\delta \ll 2 \Upsilon_{\mathrm{BBU}} /\left(k_{\beta} L_{0}\right)$.

Solution: The solution to Eq. (89) is [39]

$$
\begin{aligned}
x_{2}(s)= & \frac{\theta}{\left(k_{\beta}+\Delta k_{\beta}\right) \sqrt{1+\delta_{2}}} \sin \frac{\left(k_{\beta}+\Delta k_{\beta}\right) s}{\sqrt{1+\delta_{2}}}-\frac{2 \Upsilon_{\mathrm{BBU}} \theta}{k_{\beta} L\left(1+\delta_{2}-\left(1+\frac{\Delta k_{\beta}}{k_{\beta}}\right)^{2}\right)} \times \\
& {\left[\frac{1}{k_{\beta}} \sin k_{\beta} s-\frac{\sqrt{1+\delta_{2}}}{k_{\beta}+\Delta k_{\beta}} \sin \frac{\left(k_{\beta}+\Delta k_{\beta}\right) s}{\sqrt{1+\delta_{2}}}\right] . }
\end{aligned}
$$

Eliminating $\left(k_{\beta}+\Delta k_{\beta}\right)$ using the BNS condition, Eq. (90), this solution simplifies to [39]

$$
\begin{aligned}
x_{2}(s)= & x_{1}(s)+\frac{\theta \delta_{2}}{k_{\beta}\left(\delta_{2}-\frac{2 \Upsilon_{\mathrm{BBU}}}{k_{\beta} L}\right)}\left[\sqrt{\frac{1+\frac{2 \Upsilon_{\mathrm{BBU}}}{k_{\beta} L}}{1+\delta_{2}}} \sin \left(k_{\beta} s \sqrt{\frac{1+\frac{2 \Upsilon_{\mathrm{BBU}}}{k_{\beta} L}}{1+\delta_{2}}}\right)\right. \\
& \left.-\sin k_{\beta} s\right]=x_{1}(s)+D_{2}(s) \delta_{2}+\mathcal{O}\left(\delta_{2}^{2}\right)
\end{aligned}
$$

with

$$
D_{2}(s)=-\frac{\theta L}{2 \Upsilon_{\mathrm{BBU}}}\left[\sin \left(k_{\beta} s+\frac{\Upsilon_{\mathrm{BBU}}}{L} s\right)-\sin k_{\beta} s\right] .
$$

Note that with BNS damping the tail particle experiences no regular dispersion, and the wakeinduced dispersion is suppressed by a factor $1 / \Upsilon_{\mathrm{BBU}}$. The solution is illustrated in Fig. 8.

(5) Dispersion from a misaligned structure. The kick $\theta$ discussed above affected both head and tail particles. If a structure, e.g., an rf accelerating cavity, is misaligned, only the tail receives a kick $\theta_{s}$ while the head orbit is unperturbed, and we can write the centroid motion of head and tail as $x_{1}(s)=0$, and $x_{2}(s)=\frac{\theta_{s}}{k_{\beta}} \sin k_{\beta} s$, assuming that the centroid momentum errors are zero. Orbit correction will apply a kick $\theta=-\theta_{s} / 2$ to both the head and tail particles in order to correct the centroid motion.

Compute the dispersion for a particle in the bunch tail after orbit correction, assuming that the $B N S$ condition is fulfilled. Compare the result with the dispersion generated by a $\pi$ bump through a misaligned quadrupole given by Eq. (63).

Solution: After correcting the centroid orbit behind the misaligned structure, the particle motion for the head and tail of the bunch is roughly given by (see Eq. (60)):

$$
\begin{aligned}
& x_{1}(s) \approx-\frac{\theta_{s}}{2 k_{\beta} \sqrt{1+\delta_{1}}} \sin \frac{k_{\beta} s}{\sqrt{1+\delta_{1}}} \\
& x_{2}(s) \approx \frac{\theta_{s}}{k_{\beta} \sqrt{1+\delta_{2}}} \sin \frac{k_{\beta} s}{\sqrt{1+\delta_{2}}}-\frac{\theta_{s}}{2 k_{\beta} \sqrt{1+\delta_{2}}} \sin k_{\beta} s .
\end{aligned}
$$

Dispersion generated by the second term in the equation for $x_{2}$ will not be significant if the BNS condition is fulfilled; see Eq. (93). Thus the dispersion in the tail comes mainly from the first term 


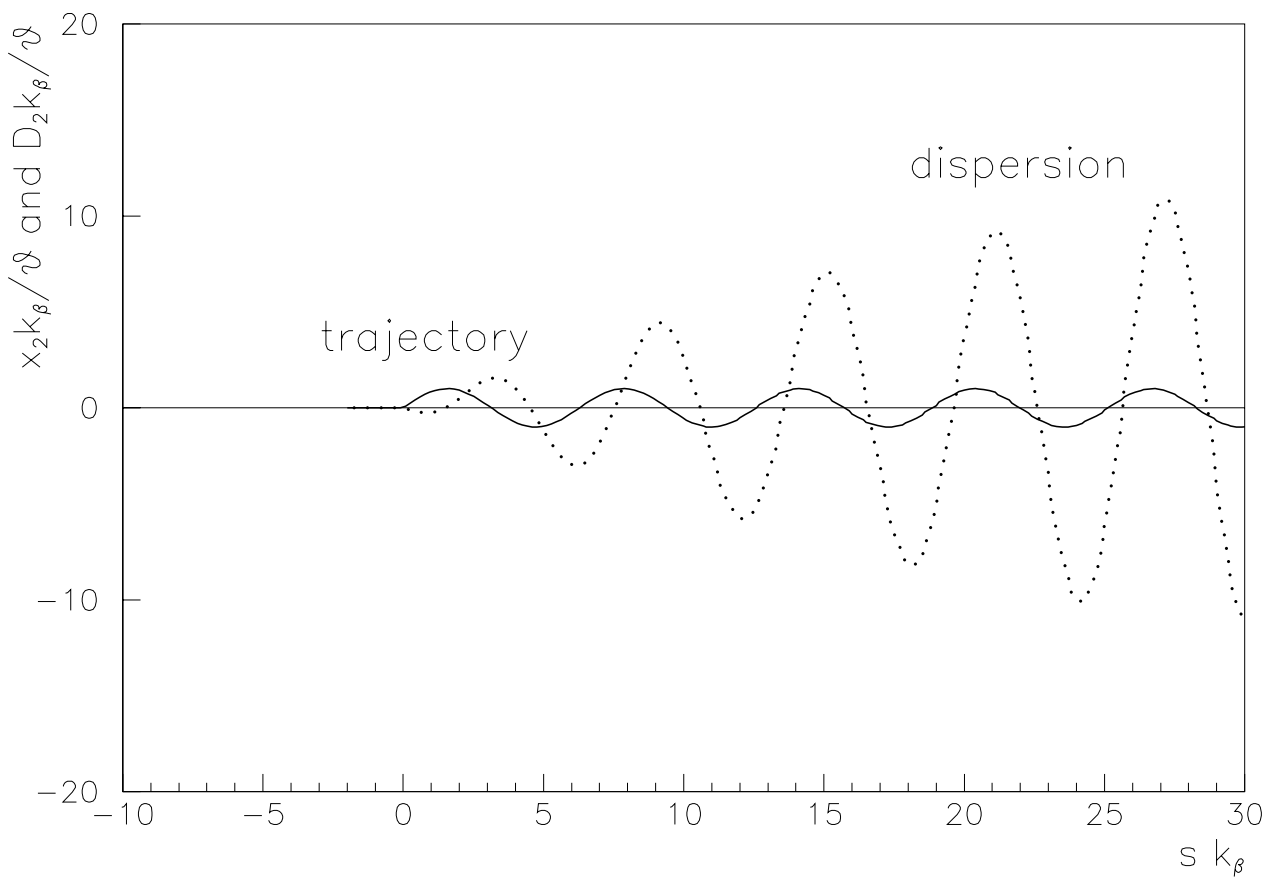

Figure 8: Oscillation of the trailing macroparticle, $x_{2} k_{\beta} / \theta$, with wake fields and BNS damping, and the tail dispersion, $D_{2} k_{\beta} / \theta$, induced by a deflection at $s=0$, according to Eqs. (92) and (93), for $\Upsilon_{\mathrm{BBU}} /\left(k_{\beta} L\right)=0.08$ (SLC value).

on the right hand side, which represents the deflection by the wake field. It is approximately given by Eq. (62) with $\theta=\theta_{s} / 2$ [39]:

$$
D_{2}(s) \approx-\frac{\theta_{s}}{4}\left[s \cos k_{\beta} s+\frac{1}{k_{\beta}} \sin k_{\beta} s\right] .
$$

The dispersion for the head particles is also obtained from Eq. (62), but with $\theta=-\theta_{s} / 2$. It thus is of the same magnitude, but of opposite sign: $D_{1}(s)=-D_{2}(s)$. Therefore, even after perfectly steering the orbit through the centre of all BPMs and quadrupoles, there can still be significant dispersion across the bunch. This solution is illustrated in Fig. 9.

\subsection{Quadrupole and Structure Misalignment}

Note that, for a quadrupole misalignment, orbit correction via $\pi$ bumps leads to a constant residual dispersion, whereas in case of a misaligned structure the orbit is corrected essentially by a single deflection and as a result the dispersion grows resonantly.

We can evaluate the magnitude of the deflection which is expected from a single structure misalignment, using again the two-particle model. Traversing a structure which is misaligned by $\Delta x_{\text {acc }}$, the tail will experience a kick

$$
\theta_{s}=\frac{N_{b} r_{e}\left(W_{1} l_{\mathrm{acc}}\right)}{2 \gamma} \Delta x_{\mathrm{acc}}
$$




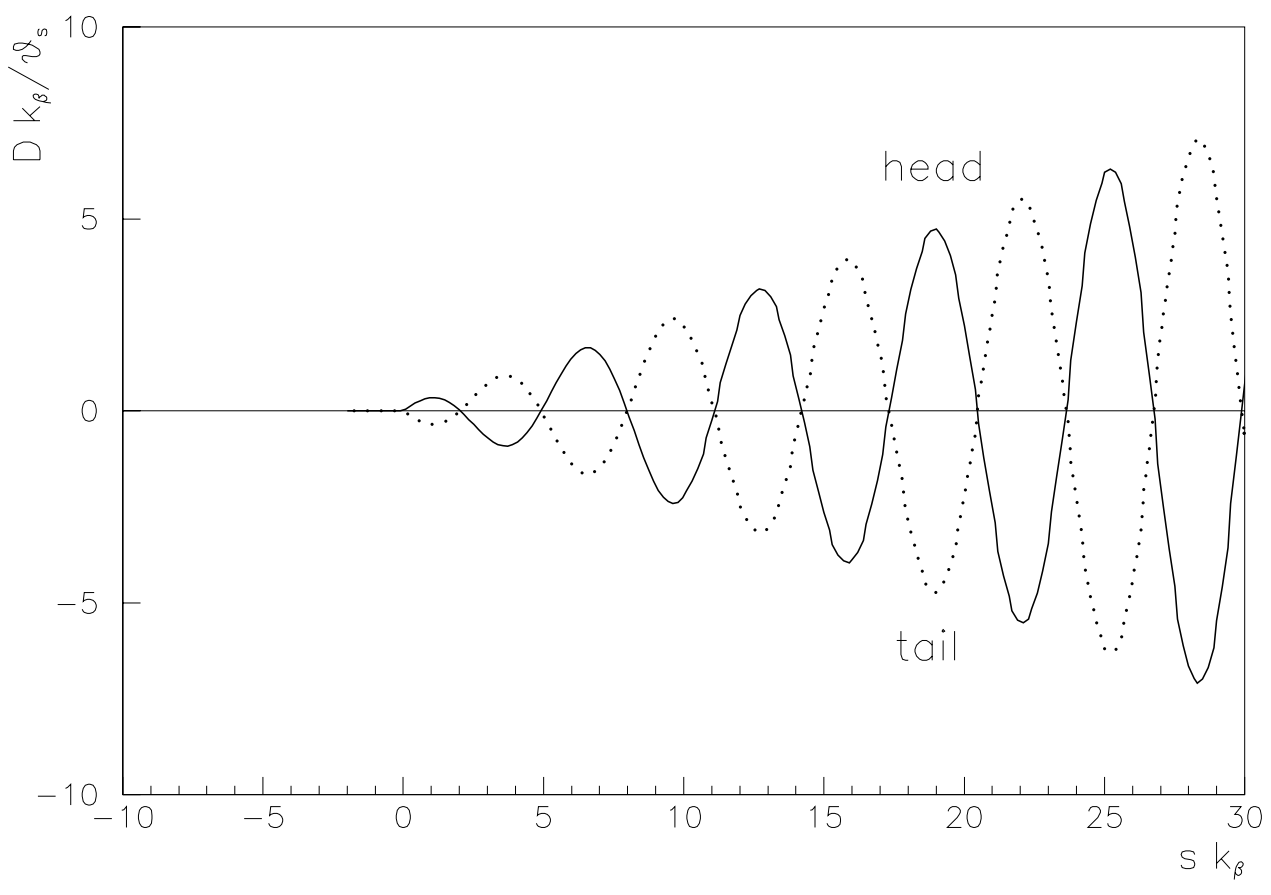

Figure 9: Dispersion for particles in bunch head and tail, $D k_{\beta} / \theta_{s}$, induced by a misaligned structure at $s=0$ deflecting the tail by $\theta_{s}$ and subsequent orbit correction, according to Eq. (96). The dispersion functions at the head and the tail are of opposite sign and both grow linearly along the linac, after Ref. [43].

where $l_{\text {acc }}$ denotes the length of the misaligned accelerator structure, and $W_{1}$ the transverse wake field per unit length in the structure, at a distance of $1-2 \sigma_{z}$. With $\gamma=2 \times 10^{4}, N=4 \times 10^{9}$, $W_{1} \approx 4 \times 10^{6} \mathrm{~m}^{-3}, l_{\text {acc }}=0.5 \mathrm{~m}$, we find $\theta_{s} \approx 6 \times 10^{-4} \Delta x_{\text {acc }}[\mathrm{m}]$.

On the other hand, approximating the integrated quadrupole strength as $K_{q} \approx 2 / \beta$, for a quadrupole misaligned by $\Delta x_{\text {quad }}$ we estimate the deflection from a displaced quadrupole as

$$
\theta=K_{q} \Delta x_{\text {quad }} \approx \frac{2}{\beta} \Delta x_{\text {quad }},
$$

or $\theta \approx 2 \times 10^{-1} \Delta x_{\text {quad }}[\mathrm{m}]$. For equal displacement $\left(\Delta x_{\text {acc }}=\Delta x_{\text {quad }}\right)$, the deflections from a quadrupole are 300 times stronger.

\subsection{Dispersion-Free Steering}

To detect and correct the residual dispersion and the wake field effects, special steering algorithms have been developed. The main idea is that not only the nominal orbit is corrected, but simultaneously also the orbits measured for different bunch charges, bunch lengths or linac-quadrupole strengths. Since the orbit difference is proportional to the dispersion or wake-field effects, the latter are then minimized as well.

One of the most important and fruitful algorithms is the dispersion-free steering [46]. Look again 
at the equation of betatron motion in the linac:

$$
x^{\prime \prime}+\frac{K}{1+\delta} x=\sum_{i} \frac{K_{i} \Delta x_{i}}{1+\delta} \delta\left(s-s_{i}\right),
$$

where on the left we used a smooth approximation for the focusing, and on the right we introduced the deflections from off-centre quadrupoles. The displacement of the quadrupole with respect to the nominal beam position is denoted $\Delta x_{i}$.

If we change the strength of all quadrupoles by $\Delta K$, or $\Delta K_{i}$, the on-momentum trajectory $(\delta=0)$ becomes

$$
x^{\prime \prime}+K\left(1+\frac{\Delta K}{K}\right) x=\sum_{i}\left(1+\frac{\Delta K_{i}}{K_{i}}\right) \Delta x_{i} \delta\left(s-s_{i}\right) .
$$

We observe that Eq. (99) for a particle trajectory with $\delta \neq 0$, is formally identical to Eq. (100) for a trajectory with $\delta=0$ and modified quadrupole strength

$$
\frac{\Delta K}{K}=\frac{\delta}{1+\delta}
$$

The idea of dispersion free steering is then, rather than to change the beam energy, to vary the relative strength of all quadrupoles, and to minimize the resulting change in beam orbit. In addition, as for conventional orbit correction also the absolute orbit readings are reduced.

The principle and benefit of dispersion-free steering is illustrated schematically in Fig. 10 [43]. Standard orbit correction will steer the beam through the centre of all BPMs, which are assumed to be fixed to the quadrupole centres. A misaligned quadrupole will provoke a bump. Although the orbit perturbation is localized, this bump will generate dispersion. The dispersive trajectory becomes visible as a change in beam orbit, when the strengths of all dipole and quadrupole correctors are scaled. Subsequent minimization of the difference trajectory results in a more efficient compensation of the misalignment, which no longer generates dispersion.

In the following we briefly outline the mathematical algorithm of dispersion-free steering [43]. The orbit generated at the $j$ th BPM by all upstream deflections $\theta_{i}$ (due to misalignments and steering coils) is

$$
x^{j}=\sum_{i=1}^{j-1} R_{12}^{i \rightarrow j} \theta_{i}
$$

where

$$
R_{12}^{i \rightarrow j}=\sqrt{\frac{E^{i}}{E^{j}}} \sqrt{\beta_{x}^{i} \beta_{x}^{j}} \sin \left[\psi_{x}^{i}-\psi_{x}^{j}\right]
$$

and we used the beam energies, beta functions, and betatron phases at corrector $i$ and monitor $j$.

Scaling all quadrupoles and dipoles in the lattice by $\kappa=1+\Delta K / K$ results in the orbit change

$$
\Delta x^{j}(\kappa)=x^{j}(0)-x^{j}(\kappa)=\sum_{i=1}^{j-1}\left(R_{12}^{i \rightarrow j}-R_{12, \kappa}^{i \rightarrow j}\right) \theta_{i}
$$

where $R_{12, \kappa}^{i \rightarrow j}$ denotes the $R$ matrix for the scaled lattice. 


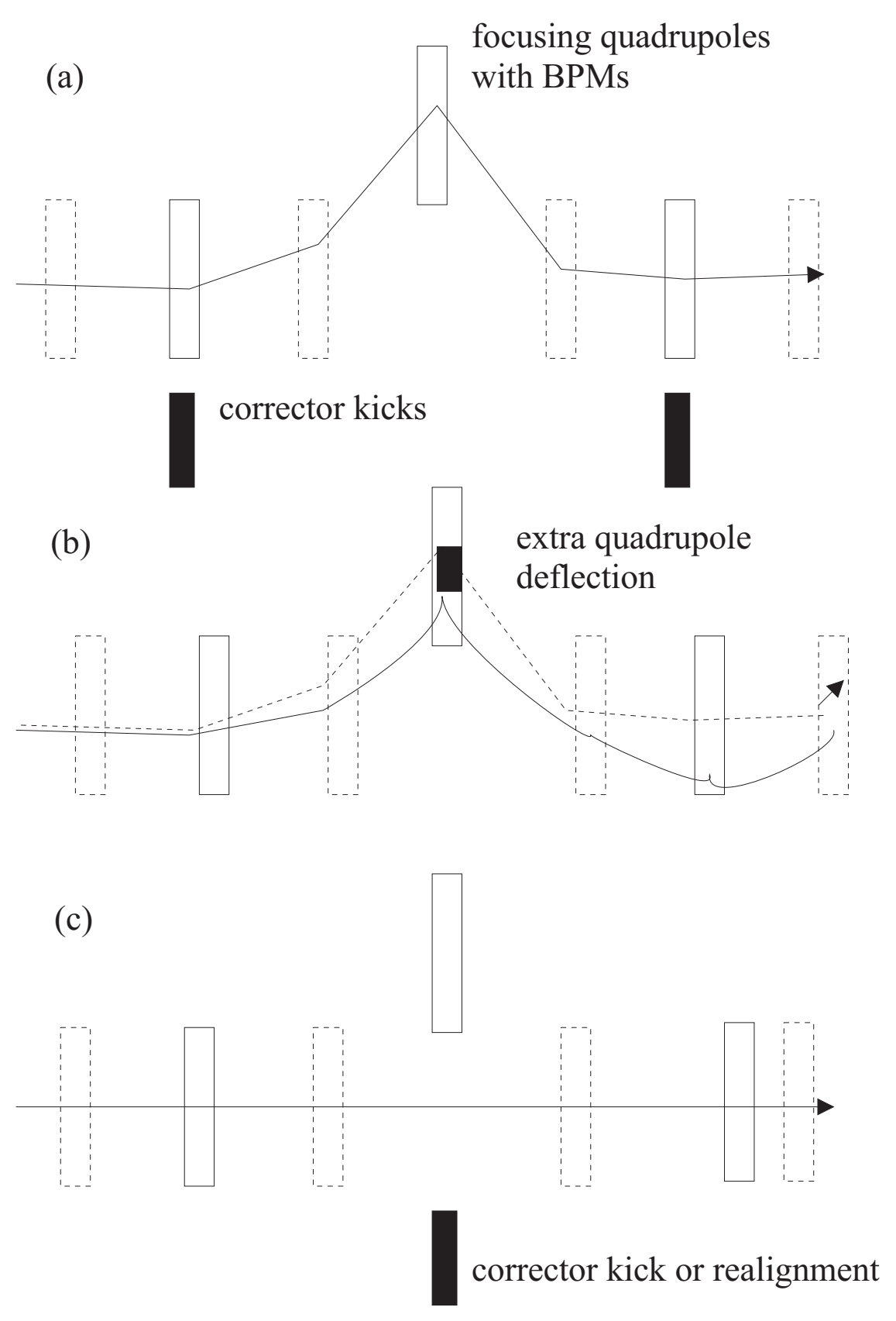

Figure 10: Schematic of dispersion-free steering, after Ref. [43]: (a) standard orbit correction which minimizes BPM readings at misaligned quadrupole thereby generating dispersion; (b) an extra deflection induced by the misaligned quadrupole which becomes apparent when quadrupoles and dipole correctors are scaled; (c) the result of the dispersion-free correction, which eliminates or compensates the kick from the misaligned quadrupoles. 
We introduce vectors for the BPM measurements and the associated weights:

$$
\vec{B}=\left(\begin{array}{c}
x^{1} \\
\Delta x^{1}\left(\kappa_{1}\right) \\
\Delta x^{1}\left(\kappa_{2}\right) \\
\Delta x^{1}\left(\kappa_{3}\right) \\
x^{2} \\
\Delta x^{2}\left(\kappa_{1}\right) \\
\Delta x^{2}\left(\kappa_{2}\right) \\
\Delta x^{2}\left(\kappa_{3}\right) \\
\cdots \\
\Delta x^{n}\left(\kappa_{3}\right)
\end{array}\right) \text { and } \vec{W}=\left(\begin{array}{c}
W^{1} \\
\Delta W_{\Delta}^{1}\left(\kappa_{1}\right) \\
\Delta W_{\Delta}^{1}\left(\kappa_{2}\right) \\
\Delta W_{\Delta}^{1}\left(\kappa_{3}\right) \\
W^{2} \\
\Delta W_{\Delta}^{2}\left(\kappa_{1}\right) \\
\Delta W_{\Delta}^{2}\left(\kappa_{2}\right) \\
\Delta W_{\Delta}^{2}\left(\kappa_{3}\right) \\
\ldots \\
\Delta W_{\Delta}^{n}\left(\kappa_{3}\right)
\end{array}\right) .
$$

The weights for the nominal orbit are a combination of the statistical variation, $\sigma^{2}\left(x^{j}\right)$, and the BPM misalignment or electronic offset, $\sigma_{\mathrm{BPM}}^{2}$ :

$$
W^{j}=\frac{1}{\sigma^{2}\left(x^{j}\right)+\sigma_{\mathrm{BPM}}^{2}} .
$$

The weight for the difference orbit includes the statistical contributions, $\sigma^{2}\left(x^{j}\right)$ and $\sigma^{2}(x j, \kappa)$, for the two orbits whose difference is computed, as well as a systematic error representing orbit or BPM drifts between the measurements, $\sigma_{\text {sys }}^{2}$ :

$$
W_{\Delta}^{j}\left(\kappa_{i}\right)=\frac{1}{\sigma^{2}\left(x^{j}\right)+\sigma^{2}\left(x^{j}, \kappa_{i}\right)+\sigma_{\mathrm{sys}}^{2}} .
$$

After averaging over many orbits the statistical contributions become negligible and the $\chi^{2}$ of the measurement reads

$$
\chi^{2} \approx \sum_{j}\left[\frac{x^{j^{2}}}{\sigma_{\mathrm{BPM}}^{2}}+\sum_{\kappa_{i}} \frac{\Delta x^{j^{2}}\left(\kappa_{i}\right)}{\sigma_{\mathrm{sys}}^{2}}\right] .
$$

Finally we define a correlation matrix

$$
\mathbf{A}=\left(\begin{array}{cccc}
R_{12}^{1 \rightarrow 1} & 0 & \ldots & 0 \\
R_{12, \kappa_{1}}^{1 \rightarrow} & 0 & \ldots & 0 \\
R_{12,1}^{1 \rightarrow \kappa_{2}} & 0 & \ldots & 0 \\
R_{12,1}^{1 \rightarrow \kappa_{3}} & 0 & \ldots & 0 \\
R_{12}^{1 \rightarrow 2} & R_{12}^{2 \rightarrow 2} & \ldots & 0 \\
R_{12, \kappa_{1}}^{1 \rightarrow 2} & R_{12, \kappa_{1}}^{2 \rightarrow} & \ldots & 0 \\
\ldots & & &
\end{array}\right)
$$

where the $R_{12} \mathrm{~s}$ are the transport-matrix elements between the correctors and BPMs. The dispersionfree trajectory is obtained by solving for the vector $\vec{X}$ of corrector settings which simultaneously minimizes the trajectory offsets and the dispersion:

$$
\min _{X}\|\vec{W}(\vec{B}+\mathbf{A} \vec{X})\|_{2}
$$

Several variations of this method have been tried successfully at the SLC. For example, instead of changing the strength of the quadrupoles, one could compare the orbits of electron and positron beams (the opposite charge is equivalent to a $-200 \%$ change in focusing strength), and the leastsquare minimization was replaced by a singular-value decomposition [47, 48]. In addition, it is possible to extend the dispersion-free algorithm so that it also minimizes the wake-field effect for coherent betatron oscillations [49]. 


\subsection{Computer Simulations}

Complementing the analytical treatment described above, dedicated computer programmes like LIAR [50], MUSTAFA [51], or PLACET [52] can provide improved estimates of emittance growth and beam stability in linear-collider linacs. These computer simulations include acceleration, magnet and structure misalignments, BPM errors, realistic steering procedures, correction algorithms, orbitfeedback systems, and both single and multi-bunch wake fields. It is reassuring that the measured emittance growth along the SLAC linac was reproduced in a simulation [53].

\section{Damping Rings}

The purpose of the damping rings is to reduce the phase space volume of a beam produced by a positron source or an electron gun so that the design beam emittances are obtained. The transverse emittances typically must be decreased by several orders of magnitude. The damping ring accomplishes this via radiation damping. The latter is characterized primarily by two parameters: the damping time and the equilibrium emittance.

\subsection{Synchrotron Radiation}

The equation describing the horizontal emittance evolution in the damping ring is

$$
\tau_{x} \frac{d \epsilon_{x}}{d t}=-2\left(\epsilon_{x}-\epsilon_{x, \infty}\right)
$$

with the solution

$$
\epsilon_{x}(t)=\epsilon_{x, 0} e^{-2 t / \tau_{x}}+\epsilon_{x, \infty}\left(1-e^{-2 t / \tau_{x}}\right)
$$

where $\tau_{x}$ is the horizontal radiation damping time, $\epsilon_{x, 0}$ the initial emittance of the injected beam and and $\epsilon_{x, \infty}$ the equilibrium emittance. If the ring is large enough, several bunch trains can be stored simultaneously, and an individual bunch train may stay in the ring for a correspondingly longer time. What matters for the performance is the effective damping time $\tau_{\text {eff }}=\tau_{x} / n_{\text {train }}$ with $n_{\text {train }}$ denoting the number of trains stored. If the effective damping time is $1 /\left(3 f_{\text {rep }}\right)$, or smaller,-where $f_{\text {rep }}$ is the repetition rate of the collider,- the initial emittance can be reduced by a factor $e^{-6} \approx 0.002$ (depending on the values of $\epsilon_{x, 0}$ and $\epsilon_{x, \infty}$ ), or more.

The expression for the horizontal damping time is

$$
\tau_{x}=\frac{1}{C_{d} J_{x} E^{3}\left\langle 1 / \rho^{2}\right\rangle}
$$

where $E$ is the beam energy, $\rho$ the bending radius, $J_{x} \approx 1$ the damping partition number, and $C_{d}=c r_{0} /\left(3 m_{e}^{3} c^{6}\right) \approx 2 \times 10^{3} \mathrm{~m}^{2} \mathrm{GeV}^{-3} \mathrm{~s}^{-1}$, where $m_{e} \approx 9.11 \times 10^{-31} \mathrm{~kg}$ denotes the electron mass. For example, with $E=2 \mathrm{GeV}$ and $\rho \approx 10 \mathrm{~m}$, we find $\tau_{x} \approx 6 \mathrm{~ms}$. We will see that for obtaining smaller equilibrium emittances it is desirable to increase the bending radius, and, hence, the circumference. In order to keep the effective damping time $\tau_{\text {eff }} \propto \rho / E^{3}$ constant, the beam energy then must also be increased as $E \propto \rho^{1 / 3}$. This constraint could be relaxed if most of the energy loss occurs in special damping wigglers and not in the arcs [54]. We will not make that assumption.

After a sufficiently long store time the beam loses the memory of the injection conditions, and its emittance acquires an equilibrium value $[20,55]$

$$
\epsilon_{x, \infty}=\frac{C_{q}}{J_{x}} \gamma^{2} \theta^{3} F
$$


where $\theta$ denotes the bending angle per dipole, assuming all dipoles are identical, and $C_{q}=3.83 \times$ $10^{-13} \mathrm{~m}$. The function $F$ is determined by the lattice,

$$
F \equiv \frac{\rho^{2}}{l^{3}}\langle\mathcal{H}\rangle_{\rho}
$$

with $l$ the length of a dipole, and

$$
\langle\mathcal{H}\rangle_{\rho}=\frac{1}{l} \int_{0}^{l}\left(\gamma_{x} D_{x}^{2}+2 \alpha_{x} D_{x} D_{x}^{\prime}+\beta_{x} D_{x}^{\prime 2}\right) d s .
$$

Here $D_{x}$ is the dispersion, $\beta_{x}$ the beta function, and $\alpha_{x}=-\frac{1}{2} \beta_{x}^{\prime}$, and $\gamma_{x}=\left(1+\alpha_{x}^{2}\right) / \beta_{x}$. In the following exercise we drop the subindex $x$. The normalized emittance, $\gamma \epsilon_{\infty}$, increases as the third power of energy and bending angle. If the length per cell is held constant, and assuming $E \propto \rho^{1 / 3}$, so as to maintain a constant effective damping time, the normalized emittance decreases inversely with the square of the radius, i.e., $\gamma \epsilon \propto \rho \theta^{3} \propto 1 / \rho^{2}$, where we considered $F \approx$ constant and $\theta \propto 1 / \rho$.

\section{Case Study IV: Minimum Emittance Lattice}

Assume that $D=D^{\prime}=0$ at one end of a dipole, so as to produce a dispersion-free straight section, and that the beta function has a minimum $\beta_{0}$ at some position $s_{0}$. Determine the values of $\beta_{0}$ and $s_{0}$ which minimize the function $F$. Recall that inside the dipole magnet the equations describing beta function and dispersion are $2 \beta \beta^{\prime \prime}-\beta^{\prime 2}-4=0$, and $D^{\prime \prime}=1 / \rho$.

Solution: the general form of Twiss parameters and dispersion functions in a region without quadrupoles and with bending radius $\rho$ (ignoring the weak focusing from the dipole) is

$$
\begin{aligned}
\beta & =\beta_{0}+\frac{\left(s-s_{0}\right)^{2}}{\beta_{0}} \\
\alpha & =-\frac{1}{2} \beta^{\prime} \\
\gamma & =\frac{1}{\beta_{0}} \\
D^{\prime} & =\frac{s}{\rho} \\
D & =\frac{1}{2} \frac{s^{2}}{\rho}
\end{aligned}
$$

Inserting this into Eq. (116), we find [55]

$$
\begin{aligned}
\langle\mathcal{H}\rangle_{\rho} & =\frac{1}{l} \int_{0}^{l}\left(\frac{1}{\beta_{0}} \frac{s^{4}}{4 \rho^{2}}-\frac{\left(2-s_{0}\right)}{\beta_{0}} \frac{s^{3}}{\rho^{2}}+\frac{\left(s-s_{0}\right)^{2}}{\beta_{0}} \frac{s^{2}}{\rho^{2}}+\beta_{0} \frac{s^{2}}{\rho^{2}}\right) d s \\
& =\frac{1}{3} \frac{l^{3}}{\rho^{2}}\left[\frac{\beta_{0}}{l}+\frac{l}{\beta_{0}}\left(\frac{s_{0}^{2}}{l^{2}}-\frac{3}{4} \frac{s_{0}}{l}+\frac{3}{20}\right)\right] .
\end{aligned}
$$

This expression becomes minimum for $s_{0} / l=3 / 8$ and $\beta_{0} / l=\sqrt{3 / 320}$, where $F=0.065$ [55].

As an example, consider a damping ring accommodating 100 bending magnets, each with angle $\theta=2 \pi / 100 \approx 0.06 \mathrm{rad}$, length $l=0.6 \mathrm{~m}, \rho=10 \mathrm{~m}$, and $E=2 \mathrm{GeV}(\gamma \approx 4000)$. For $F=0.065$ the equilibrium emittance would be

$$
\gamma \epsilon_{x} \approx 3.4 \times 10^{-7} \mathrm{~m}
$$


Table 2: Example damping ring parameters for 3-TeV linear collider, scaled from the 1-TeV lattice of Ref. [56]

\begin{tabular}{|lcc|}
\hline variable & symbol & value \\
\hline energy & $E$ & $3.0 \mathrm{GeV}$ \\
circumference & $C$ & $700 \mathrm{~m}$ \\
hor./vert. emit. & $\gamma \epsilon_{x, y}$ & $5 / 0.1 \times 10^{-7} \mathrm{~m}$ \\
hor. beam size & $\sigma_{x}$ & $30 \mu \mathrm{m}$ \\
vert. beam size & $\sigma_{y}$ & $4 \mu \mathrm{m}$ \\
hor./vert. half ap. & $h_{x, y}$ & $2 \mathrm{~cm}$ \\
av. beta function & $\beta_{x, y}$ & $\sim 10 \mathrm{~m}$ \\
bunch length & $\sigma_{z}$ & $1.8 \mathrm{~mm}$ \\
train length & $l_{\text {train }}$ & $154 \mathrm{~ns}$ \\
no. of bunches & $n_{b}$ & 154 \\
bunch population & $N_{b}$ & $4 \times 10^{9}$ \\
bunch spacing & $L_{\mathrm{sep}}$ & $0.2 \mathrm{~m}$ \\
\hline
\end{tabular}

close to the requirements of a future multi-TeV collider (see Table 1).

However, in practice it is not easy to design a lattice with the optimum parameters calculated above. For a more realistic lattice, composed of a symmetric achromatic bend connecting two dispersion-free straight sections, the minimum value of $F$ turns out to be 0.1054 [55]. Then, in order to still achieve the desired horizontal emittance, in our example the number of bending magnets must be increased by a factor $(0.1054 / 0.065)^{1 / 3}$, or $20 \%$, namely from 100 to 120 .

Table 2 lists a crudely scaled tentative parameter set for the damping ring of a 3-TeV CLIC collider. Later on we will use these parameters for estimating various instability growth rates. Note that the rms bunch length in the ring is a few millimeters, whereas in the linac and at the collision point bunch lengths of the order $30-150 \mu \mathrm{m}$ are required. A similar situation is encountered for NLC and TESLA. Therefore, all linear-collider designs foresee a bunch compression after extraction from the damping ring and prior to injection into the main linac.

\subsection{Intrabeam Scattering}

Hitherto, we have calculated only the ideal horizontal emittance due to synchrotron radiation alone. For small beam sizes, multiple scattering of particles inside the bunch off each other will increase all three emittances. This effect is known as 'intrabeam scattering'.

The equilibrium emittance with intrabeam scattering can be obtained from a balance of two excitations and one damping term as [57]

$$
\epsilon_{x}=\frac{1}{4} \tau_{x}\left[Q_{x}^{S R}+Q_{x}^{I B S}\right]
$$

where

$$
Q_{x}^{S R}=\frac{55}{24 \sqrt{3}} \frac{r_{e}^{2} c}{\alpha} \gamma^{5}\left\langle\mathcal{H}_{x} / \rho^{3}\right\rangle
$$

refers to the quantum excitation and

$$
Q_{x}^{I B S}=\left\langle\frac{N r_{e}^{2} c \beta_{x}}{8 \pi \gamma^{3} \sigma_{x}^{2} \sigma_{y} \sigma_{z}} f\left(\chi_{m}\right) \mathcal{H}_{x}\right\rangle
$$


to the intrabeam scattering. The latter occurs all around the ring, whereas the former comes only from the dipoles (since $1 / \rho \approx 0$ at other places, if the beam has no large offsets in the quadrupoles). In Eq. (127) we used the following symbols [57]: $\chi_{m}=r_{e} \beta_{x}^{2} /\left(b_{\max } \gamma^{2} \sigma_{x}^{2}\right)$, $b_{\max }=\left(N /\left((2 \pi)^{3 / 2} \sigma_{x} \sigma_{y} \sigma_{z}\right)\right)^{-1 / 3}, \mathcal{H}_{x}=\frac{1}{\beta_{x}}\left[D^{2}+\left(\beta D^{\prime}-\frac{1}{2} \beta^{\prime} D\right)^{2}\right]$, and $f\left(\chi_{m}\right) \approx 50-200$ for $\chi_{m} \approx 10^{-5}-10^{-9}$. The angular brackets indicate an average over the ring: $\langle(\ldots)\rangle \equiv 1 / C \oint(\ldots) d s$.

Combining the above expressions we obtain

$$
\epsilon_{x}=\frac{\tau_{x}}{4}\left[\frac{55}{24 \sqrt{3}} \frac{r_{e}^{2} c}{\alpha} \gamma^{5}\left\langle\frac{\mathcal{H}_{x}}{\rho^{3}}\right\rangle+\frac{N r_{e}^{2} c f\left(\chi_{m}\right)}{8 \pi \gamma^{3} \epsilon_{x}^{3 / 2} \sqrt{\kappa} \sigma_{z}}\left\langle\frac{\mathcal{H}_{x}}{\beta_{y}^{1 / 2}}\right\rangle\right]
$$

with $\kappa=\epsilon_{y} / \epsilon_{x}$, and $\sigma_{z}$ the increased bunch length [57]

$$
\sigma_{z} \approx \sigma_{z 0}\left(1+\frac{N r_{e}^{2} c \beta \tau_{\delta} f\left(\chi_{m}\right)}{\sigma_{\delta, 0}^{2} 32 \pi \gamma^{3} \sigma_{x 0}^{2} \sigma_{y 0} \sigma_{z 0}}\right)^{1 / 2}
$$

The subindex 0 denotes the beam sizes and rms energy spread without intrabeam scattering, and $\tau_{\delta}$ the longitudinal damping time.

The equilibrium emittance with intrabeam scattering can be estimated by solving Eqs. (128) and (129) numerically, or it can be calculated more accurately using computer codes such as ZAP $[58,59]$. Typically, intrabeam scattering increases the equilibrium emittance of a linear-collider damping ring by $20-50 \%$.

\subsection{Emittance Measurements}

How can one verify that the expected emittance has been achieved? The Accelerator Test Facility (ATF) [60] at KEK in Japan is a prototype damping ring, which was built to stably produce a low emittance beam as required by a future $1-\mathrm{TeV}$ linear colliders, and to develop adequate tuning techniques. Over the last few years a large part of the ATF machine studies were devoted to measuring the vertical emittance. Unlike the horizontal emittance, the vertical emittance is not determined by the design accelerator optics, but arises from residual vertical dispersion and linear coupling.

In light sources often the image from a synchrotron light monitor is used to infer the beam size $\sigma_{y}$ (or the beam divergence $\sigma_{y^{\prime}}$ ) and from this the emittance via $\epsilon_{y}=\sigma_{y}^{2} / \beta_{y}$ (or $\epsilon_{y}=\sigma_{y}^{\prime 2} \beta_{y}$ ), taking into account possible corrections for the depth of field and diffraction [61]. This approach is not directly feasible for the small emittances in the linear collider damping ring. Using standard deviations for a Gaussian distribution, the diffraction-limited photon beam emittance is $\epsilon_{\gamma} \sim \lambda_{\gamma} /(4 \pi \gamma)$, from Heisenberg's uncertainty principle, where $\lambda_{\gamma}$ denotes the photon wavelength. If no other informations are available, it is not possible by conventional imaging techniques to resolve electron beam emittances smaller than the diffraction-limited photon-beam emittance. However, often, e.g., when the photon divergence is much larger than the beam divergence, the beam emittance can still be determined from a photon image, using the known optical functions at the light emission point.

For soft $\mathrm{x}$-rays with $\lambda \approx 5 \mathrm{~nm}$ as monitored at the LBNL ALS, the photon-beam diffraction limit is reached at $\epsilon_{\gamma} \approx 400 \mathrm{pm}$, still much larger than the ATF design vertical emittance of $10 \mathrm{pm}$. Two possibilities to measure the ATF beam emittance would be x-ray imaging with compound refractive lenses (CRL) or using a pin-hole camera at sub-Angstrom wavelengths [62]. At the ESRF, CRLs provide a beam-size resolution of $4 \mu \mathrm{m}$ for a photon energy of $23 \mathrm{keV}$ [63], and an x-ray pin-hole camera may diagnose emittances down to $5 \mathrm{pm}$, at typical photon energies of $40 \mathrm{keV}$ [64].

One can also take advantage of the closeness to the diffraction limit and infer the beam size from the visibility of an interference pattern [65, 66]. At the KEK ATF a stellar interferometer is employed for this purpose. A simplified schematic is shown in Fig. 11. Quasi-monochromatic 
synchrotron light at $\lambda=500 \mathrm{~nm}$ is sent through a double slit, and the interference pattern observed on a screen behind. An ideal point source creates a perfect interference pattern. If the source has a finite vertical extent, the contrast of the interference is reduced. This can not only be used to measure the emittance, but has also proven to be a valuable online tuning tool for optimizing the emittance, e.g., by varying bumps or skew quadrupoles in the ring. The most precise emittance values are obtained by measuring the interference contrast as a function of distance between the two slits. The resolution is presently limited by vibrations of the interferometer support platform and the required exposure time (several $\mathrm{ms}$ ).

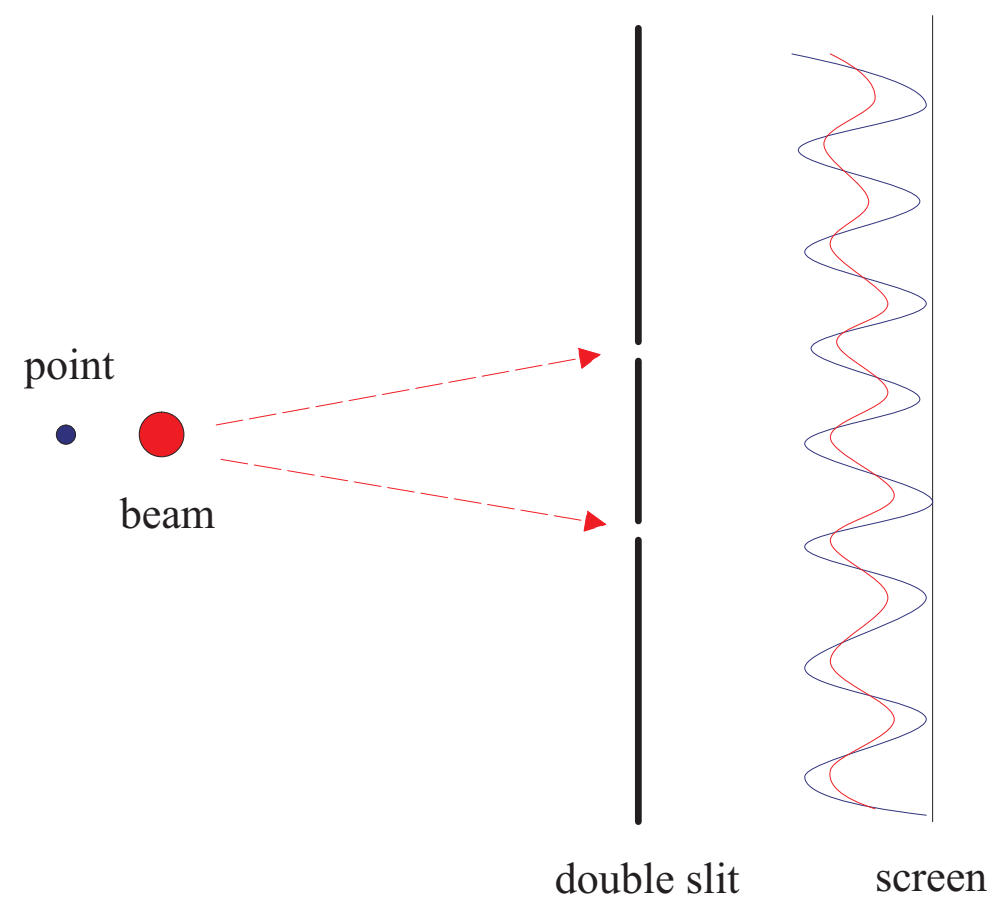

Figure 11: Schematic of ATF beam size measurement using stellar interferometer $[65,66]$.

In addition to the interferometer, the ATF team has developed three other procedures for measuring the vertical beam emittance in the damping ring.

The rms energy spread can be inferred from the beam size at a high-dispersion point, either in the ring or after extraction. Because of intrabeam scattering the energy spread increases with bunch current. The intrabeam-scattering blow up depends on the transverse and longitudinal emittances. If horizontal and longitudinal emittances are known, the vertical emittance may be obtained by fitting the measured increase in energy spread to theoretical expressions [57, 67, 68].

A similar technique can be applied to the beam lifetime, which for small emittances is limited by the Touschek effect [69]. This refers to binary collisions of particles within a bunch, by which so much energy is transferred from transverse into longitudinal phase space, that the scattered particles leave the stable rf bucket. Since it is caused by a particle-particle collision, the loss rate due to the Touschek effect is quadratic in the bunch population, and inversely proportional to the bunch volume. Again, if horizontal emittance and bunch length are known, the measured beam lifetime as a function of current can be used to estimate the vertical emittance by fitting to the analytical expressions [70]. Since the theory of the Touschek lifetime is simpler than the theory for intrabeam scattering, and since, in addition, an increase of the energy spread could also be caused by longitudinal instabilities, the lifetime method appears to be the most reliable of the two. 
The clearest verification of the ring emittance is to extract the beam from the ring and to measure the beam sizes at various wire scanners. Note that conventional wire scanners cannot be employed inside the ring, as the beam would break the wires within a few turns. Therefore, for the ATF ring an unbreakable laser wire is under development [71].

It is rather straightforward to compute the beam emittance from wire-scanner measurements. We define a reference point $s_{0}$ and denote the $R$ matrix between $s_{0}$ and wire number $i$ by $R^{(i)}$; see the illustration in Fig. 12.

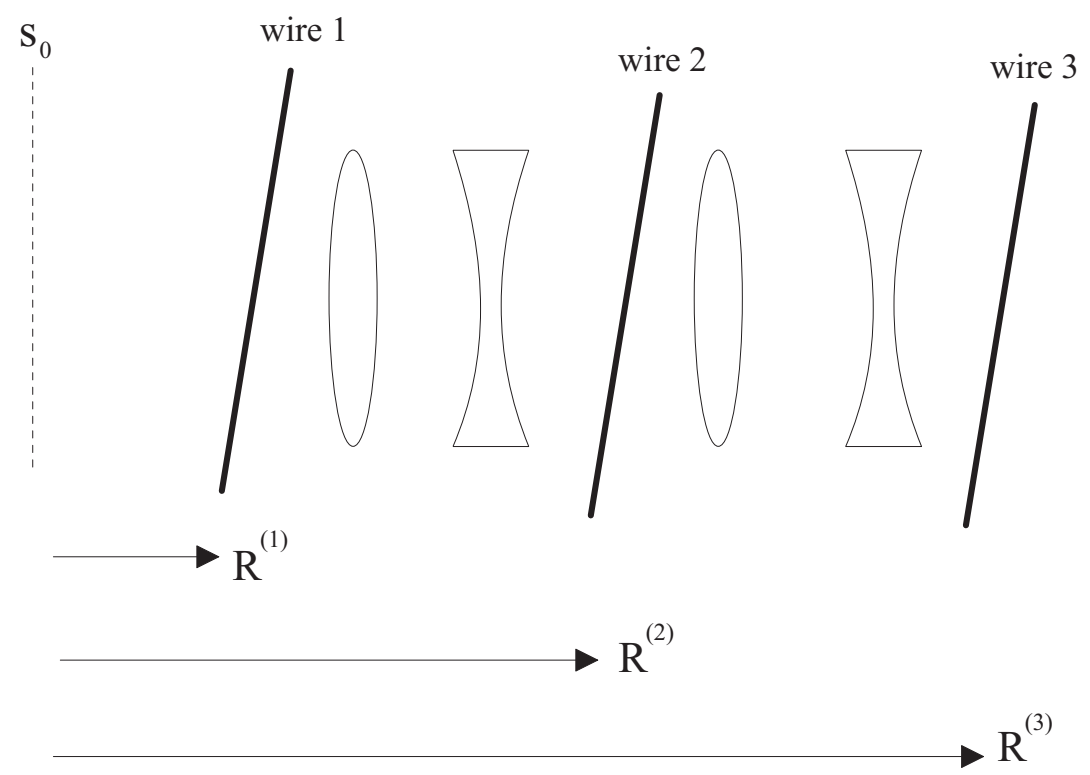

Figure 12: Schematic of emittance measurement using multiple wires.

Considering purely horizontal or vertical motion, the $R$ matrix reads

$$
R^{(i)}=\left(\begin{array}{ll}
R_{11}^{(i)} & R_{12}^{(i)} \\
R_{21}^{(i)} & R_{22}^{(i)}
\end{array}\right) .
$$

The measured squared beam sizes, the Twiss parameters at point $s_{0}$ and the emittance are related as follows:

$$
\left(\begin{array}{c}
\sigma_{x}^{(1) 2} \\
\sigma_{x}^{(2) 2} \\
\cdots \\
\sigma_{x}^{(n) 2}
\end{array}\right)=\left(\begin{array}{ccc}
R_{11}^{(1) 2} & -2 R_{11}^{(1)} R_{12}^{(i)} & R_{22}^{(1) 2} \\
R_{11}^{(2) 2} & -2 R_{11}^{(2)} R_{12}^{(i)} & R_{22}^{(2) 2} \\
\cdots & \cdots & \ldots . \\
R_{11}^{(n) 2} & -2 R_{11}^{(2)} R_{12}^{(n)} & R_{22}^{(n) 2}
\end{array}\right)\left(\begin{array}{c}
\beta_{0} \epsilon \\
-\alpha_{0} \epsilon \\
\gamma_{0} \epsilon
\end{array}\right) .
$$

The recipe is now to determine a least-square solution for the vector $\left(\beta_{0} \epsilon,-\alpha_{0} \epsilon, \gamma_{0} \epsilon\right)$, and then to solve for the initial Twiss parameters and the emittance. Note that $\gamma_{0}=\left(1+\alpha_{0}^{2}\right) / \beta_{0}$, and, hence, all variables can be calculated. Evidently, for a meaningful measurement at least 3 wire scanners at different betatron phases are required. This is called a multiwire emittance measurement.

Alternatively, if only one wire is available, one can measure the beam size at this wire for different strengths of an upstream quadrupole magnet. This is known as a quadrupole scan. Mathematically it can also be described by Eq. (131). The only difference is that now the $R$ matrices do not refer to different wires but to different settings of the quadrupole.

Timing and pulse-amplitude jitter of the extraction kicker may affect the beam stability and hence impair the quality of the wire scans, since the latter are not single-shot measurements but require of 
the order of 50 beam pulses during which the wire is moved across the beam. It took some effort to sufficiently stabilize the ATF extraction, but in spring 2000 an extremely small vertical emittance close to the design value was demonstrated with good scan quality. Figure 13 shows recent wire scans in the ATF extraction line and illustrates the high degree of stability that has been achieved.
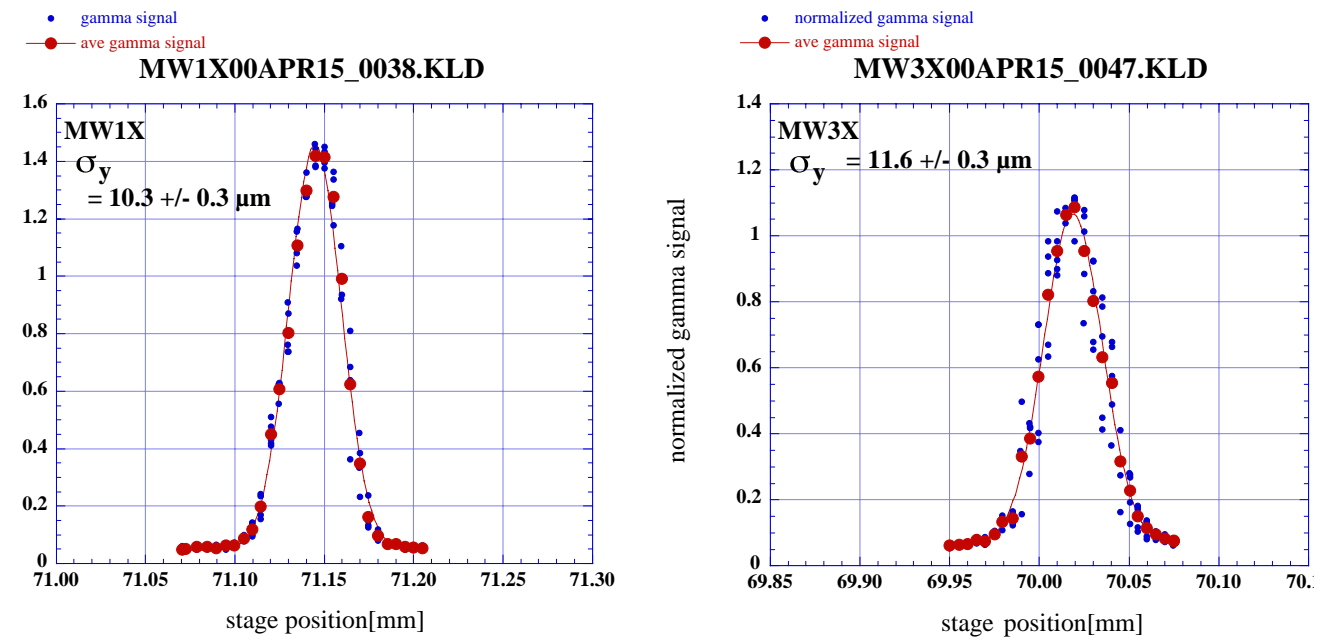

- normalized gamma sign

- ave gamma signal

MW2X00APR15_0043.KLD

- normalized gamma signal

- ave gamma signal

MW4X00APR15 0051.KLD
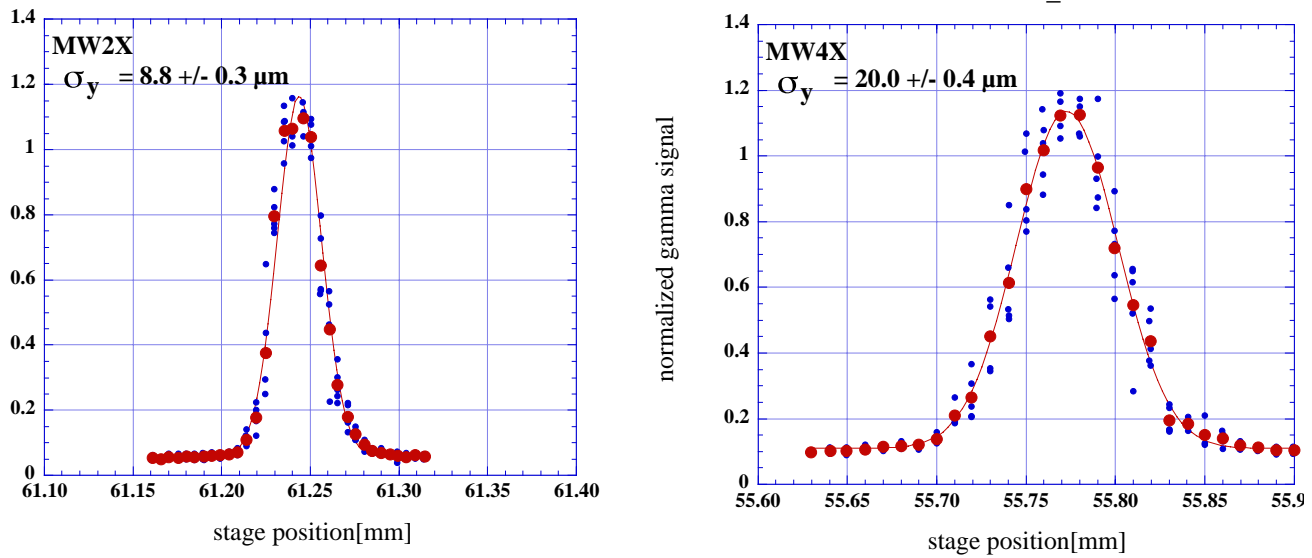

Figure 13: Vertical wire scans in the ATF extraction line, using W filaments with $10 \mu \mathrm{m}$ diameter. (Courtesy J. Urakawa.)

\subsection{Momentum Compaction Factor}

There are of course many other effects which must be carefully considered in the design of the damping rings. Some are related to the small value of the momentum compaction factor. This factor, denoted $\alpha_{C}$, quantifies the change in electron path length, $C_{e}$, as a function of relative energy deviation,

$$
\alpha_{C}=\frac{\Delta C_{e} / C_{e}}{\Delta p / p}
$$


Table 3: Momentum compaction factor for existing and proposed rings.

\begin{tabular}{|lc|}
\hline ring & $\alpha_{C}$ \\
\hline SLC & $1.8 \times 10^{-2}$ \\
ATF & $2.1 \times 10^{-3}$ \\
NLC & $4.7 \times 10^{-4}$ \\
TESLA & $3.4 \times 10^{-4}$ \\
1-TeV CLIC & $2.4 \times 10^{-4}$ \\
LEP & $1.9 \times 10^{-4}$ \\
3-TeV CLIC & $4.0 \times 10^{-5}$ \\
\hline
\end{tabular}

or, vice versa, if the rf frequency and thus the electron path length $C_{e}$ stay constant, $\alpha_{C}$ gives the change in electron momentum as a function of the circumference $C$,

$$
\Delta p / p=\frac{1}{\alpha_{C}} \frac{\Delta C}{C}
$$

The momentum compaction factor can be calculated from the dispersion function $D_{x}$ and the local bending radius $\rho$ as

$$
\alpha_{C}=\frac{1}{C} \oint \frac{D}{\rho} d s
$$

It is often convenient to approximate the momentum compaction factor as [20] $\alpha_{C} \approx 1 / \nu_{x}^{2}$, suggesting that $\alpha_{C} \propto 1 / \rho^{2}$ for constant cell length.

In future damping rings, a small equilibrium emittance is achieved by reducing the dispersion in the bending magnets. According to Eq. (134), we expect that the momentum compaction factor will also decrease. Table 3 shows that this is indeed the case. The values of $\alpha_{C}$ in future designs are two orders of magnitude smaller than at the SLC.

If the ambient temperature changes by an amount $\Delta T$, the floor and magnet supports will expand or contract by $\Delta l / l \propto \alpha_{T} \Delta T$, where $\alpha_{T}$ denotes the thermal expansion coefficient of the tunnelfloor or magnet-support material. The average path length of the electron beam is determined by the rf frequency, and unchanged by the temperature variation. However, if the quadrupole magnets move inwards or outwards due to thermal expansion, the beam will experience additional bending fields from the resulting off-centre orbit in the quadrupoles, and its energy will change.

Combining the two equations above, the beam energy change is given by

$$
\frac{\Delta p}{p}=\frac{\alpha_{T}}{\alpha_{C}} \Delta T
$$

For example, taking a typical value of $\alpha_{T} \approx 10^{-5}$, the CLIC damping ring temperature should be stabilized to $0.5 \mathrm{mK}$, in order to maintain a constant beam energy to within $10 \%$ of the rms energy spread. This tight tolerance could be relaxed by means of an automatic path-length feedback employing chicanes. Such a scheme might be tested at the ATF damping ring [72]. It is noteworthy that, despite of an extremely small momentum compaction factor, temperature variation does not appreciably affect the LEP circumference. The largest relative energy excursions at LEP, of the order of $10^{-4}$, are caused by tidal effects [73].

Aside from the increased sensitivitiy to temperature, the low momentum compaction also increases the likelihood of longitudinal single-bunch instabilities. The current threshold for the longitudinal microwave instability, with growth times much shorter than a synchrotron period, can be 
estimated from the so-called Boussard criterion [74, 75]:

$$
\frac{N e^{2} c|Z / n|}{(2 \pi)^{3 / 2} \alpha_{C} E \sigma_{\delta}^{2}} \approx 1 .
$$

The term $Z / n$ refers to the longitudinal impedance, with $Z$ denoting the impedance at $n \omega_{0}\left(\omega_{0}\right.$ is the angular revolution frequency), and $n$ the number of revolution harmonics.

Assuming a constant number and size of strong inductive impedance sources, such as rf cavities, the longitudinal impedance decreases inversely with the bending radius [12], $Z / n \propto 1 / \rho$. On the other hand, $\alpha_{C}$ decreases more strongly, as $\alpha_{C} \propto 1 / \rho^{2}$ (since $D / \rho \propto 1 / \rho^{2}$ ). Recalling the assumed energy scaling $E \propto 1 / \rho^{1 / 3}$, we can stay below the threshold, if the current per bunch decreases as $1 / \rho^{2 / 3}$.

Again, we consider some example parameters for CLIC. Using Eq. (136) with $\alpha_{C}=2.4 \times 10^{-3}$, $N=4 \times 10^{9}, \sigma_{z}=1.8 \mathrm{~mm}, E=2.15 \mathrm{GeV}, \sigma_{\delta}=8.2 \times 10^{-4}$, an upper bound for the acceptable impedance is $|Z / n|<0.05 \Omega$. This appears feasible, since for the smaller damping ring of the NLC [9] a more detailed evaluation predicts $|Z / n| \approx 0.03 \Omega$.

\subsection{Novel Instabilities}

The performance of the recently commissioned B factories, at SLAC and KEK, is affected by new types of instabilities, in which electron beams interact with ions created from the residual gas, and positron beams with photo- and secondary electrons.

\subsubsection{FAST BEAM-ION INSTABILITY}

The fast beam ion instability [76, 77] is a single-pass coupled-bunch instability occurring in electron beams; see the schematic in Fig. 14. It is driven by ions which are created from the residual gas during the single passage of a bunch train. It was first observed at the LBL ALS [78], and, shortly thereafter, confirmed at the Pohang Light Source [79]. Recently, it has been observed also at the ESRF when operated with a low-emittance optics and degraded vacuum pressure [80].

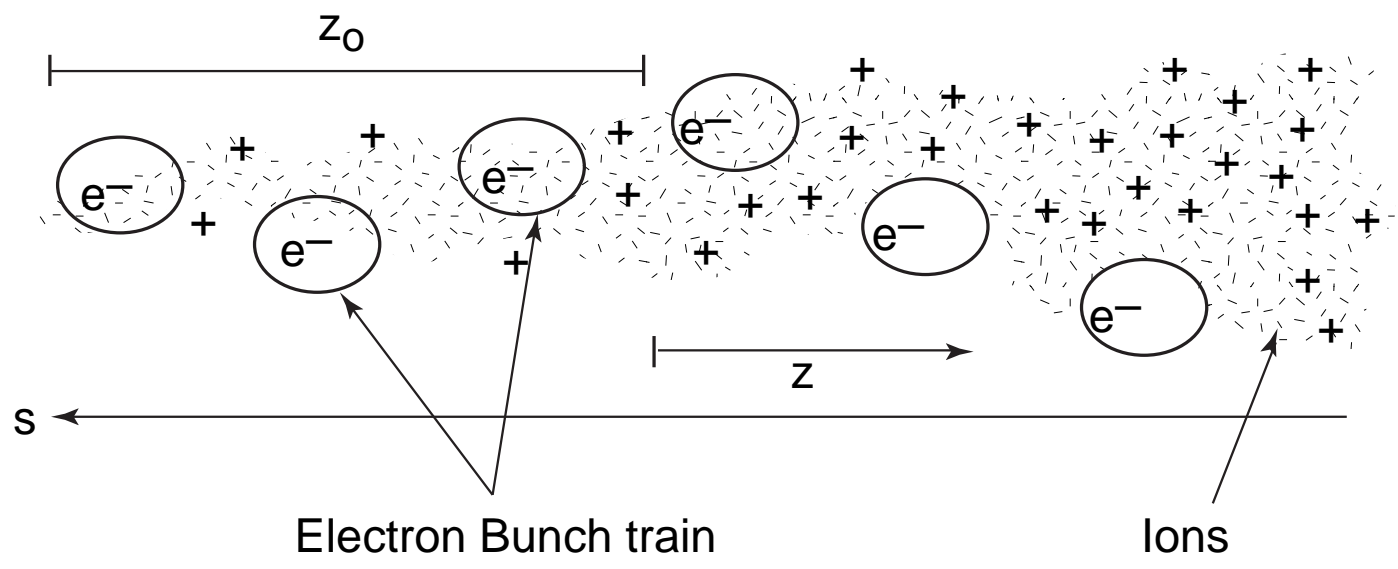

Figure 14: Schematic of fast beam-ion instability $[76,77]$, which can arise due to ion trapping in $\mathrm{e}^{-}$ bunch train.

The linear theory [76] predicts a quasi-exponential rise time, $y \propto \exp \sqrt{t / \tau_{c}}$, with a characteristic 
growth rate

$$
\frac{1}{\tau_{c}}=\frac{4 d_{\text {gas }} \sigma_{\text {ion }} \beta_{y} N_{b}^{3 / 2} n_{b}^{2} r_{e} r_{p}^{1 / 2} L_{\text {sep }}^{1 / 2} c}{\sqrt{3} 3 \gamma \sigma_{y}^{3 / 2}\left(\sigma_{x}+\sigma_{y}\right)^{3 / 2} A^{1 / 2}}
$$

at the end of the bunch train. A refined theory [81], taking into account the decoherence due to ion frequency spread within the bunch and around the ring, predicts an exponential growth, $y \propto$ $\exp \left(t / \tau_{e}\right)$, with growth rate

$$
\frac{1}{\tau_{e}} \approx \frac{1}{\tau_{c}} \frac{5 c}{\sqrt{2} l_{\text {train }} \tilde{\omega}_{i}}
$$

for the last bunch in the train, where

$$
\tilde{\omega}_{i} \equiv c\left(\frac{4 N_{b} r_{p}}{3 A L_{s e p} \sigma_{y}\left(\sigma_{x}+\sigma_{y}\right)}\right)^{\frac{1}{2}}
$$

denotes the coherent angular ion oscillation frequency, $l_{\text {train }}$ the length of the bunch train (in meters), $L_{\text {sep }}$ the bunch spacing in meters, $d_{\text {gas }}$ the gas density (in molecules per cubic meter), $n_{b}$ the number of bunches, $N_{b}$ the bunch population, $\sigma_{\text {ion }}$ the ionization cross section, and $\sigma_{x, y}$ the average horizontal and vertical rms beam sizes.

Assuming an ionization cross section of $\sigma_{\text {ion }} \approx 2$ Mbarn, e.g., for carbon monoxide, a gas density of $d_{\text {gas }} \approx 3 \times 10^{13} \mathrm{~m}^{-3}$, and an atomic mass $A=28$ (carbon monoxide or nitrogen), a pressure $p=1$ nTorr and the $3-\mathrm{TeV}$ ring parameters discussed above, we find $\tilde{\omega}_{i} \approx 7 \times 10^{8} \mathrm{~s}^{-1}, \tau_{c} \approx 1 \mu \mathrm{s}$, and $\tau_{e} \approx 20 \mu \mathrm{s}$. The latter growth rate corresponds to about 10 turns, so that a bunch-by-bunch feedback system may be effective.

\subsubsection{ELECTRON-ClOUd InSTABility}

Positron or proton beams can suffer from a different kind of instability. Because of their opposite charge they may interact with the electron cloud created by photoemission or secondary emission. Figure 15 shows a schematic of the electron cloud build up during the passage of a bunch train in the LHC beam pipe.

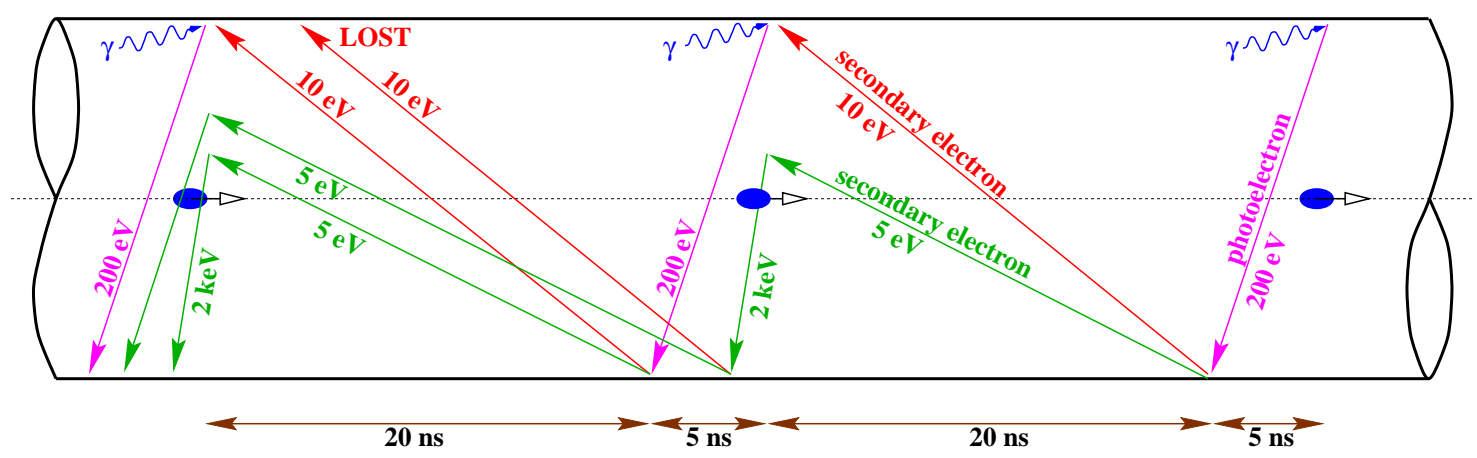

Figure 15: Schematic of electron-cloud build up in the LHC beam pipe. (Courtesy Francesco Ruggiero.)

So far two different manifestations of electron-cloud instabilities have been observed with multibunch positron beams. The so-called Ohmi effect [82] refers to a coupled-bunch instability caused by the electron cloud. This instability was first seen at the KEK photon factory $[82,83]$ and later verified at BEPC [84]. 
The second electron instability, potentially more harmful, is a head-tail instability driven by electron cloud, where the cloud produced by previous bunches acts like a short range wake field [85]. The resulting single-bunch instability was observed with positron beams at KEKB LER [86] and SLAC PEP-II, and, possibly, with the LHC proton beam at the CERN SPS [87].

We can derive a rough analytical estimate for the strength of this instability [85]. According to simulations, after a few bunches the electron cloud reaches a saturation density which is roughly equal to the neutralization density, defined as

$$
\rho_{e} \approx \frac{N_{b}}{\pi h_{x} h_{y} L_{\mathrm{sep}}} .
$$

At this density the time average of the electric field on the chamber wall vanishes. Here $h_{x}$ and $h_{y}$ are the horizontal and vertical chamber half apertures and $L_{\text {sep }}$ the bunch spacing (in meters). Note that, unlike the beam, the electrons are distributed almost uniformly across the entire vacuum chamber, and only a small portion is in the vicinity of the beam at the moment of a bunch passage. This fraction of electrons is responsible for the single-bunch instability. Considering a flat beam, and using a two-particle model (due to the peculiar nature of the electron-cloud 'wake', the head particle is not chosen to be pointlike but to have a finite longitudinal extent of the order of the bunch length), an approximately constant vertical wake function can be derived [85]:

$$
W_{1} \approx \frac{8 C}{h_{x} h_{y} L_{\mathrm{sep}}} .
$$

This transverse wake function has the remarkable property that it depends only on the vacuum chamber dimensions and on the bunch spacing.

The wake function of Eq. (141) is a good approximation as long as the bunches are longer than $\sigma_{x} \sigma_{y} /\left(N_{b} r_{e}\right)$, with $r_{e}$ the classical electron radius, and $N_{b}$ the bunch population. For short bunches the formula must be modified [85].

By inserting the wake function $W_{1}$ into the standard expressions for instability growth rates [12], we can estimate the growth rates for fast beam break up (ignoring the synchrotron motion) as

$$
\frac{1}{\tau} \approx \frac{2 \pi \rho r_{e} c\left\langle\beta_{y}\right\rangle}{\gamma}
$$

and the growth rate for the $l=1$ head-tail mode of the regular head-tail instability as

$$
\frac{1}{\tau^{(1)}} \approx \frac{64}{3} \frac{\rho\left\langle\beta_{y}\right\rangle r_{e} \sigma_{z} Q_{y}^{\prime}}{T_{0} \alpha_{C} \gamma} .
$$

In addition, we can compute the threshold density for the strong head-tail instability. It reads

$$
\rho_{\mathrm{thr}}=\frac{2 \gamma Q_{s}}{r_{e} C \beta_{y}}
$$

where $\beta_{y}$ is the average beta function, weighted with the local electron cloud density.

Using our example parameters for the positron damping ring and applying these approximate relations, the saturated electron density is about $\rho_{e \text {,neutr }} \approx 1.6 \times 10^{13} \mathrm{~m}^{-3}$, the wake function $W_{1} \approx$ $7 \times 10^{7} \mathrm{~m}^{-2}$, the growth rate for beam break up $1 / \tau_{\mathrm{BBU}} \approx 1.4 \times 10^{5} \mathrm{~s}^{-1}$, the growth rate for the $l=1$ head-tail instability $1 / \tau^{(1)} \approx 1.6 \times 10^{4} Q_{y}^{\prime} \mathrm{s}^{-1}$, and the threshold density for the strong head-tail instability (also called TMCI instability) $\rho_{\mathrm{thr}} \approx 3 \times 10^{12} \mathrm{~m}^{-3}$. Note that the estimated neutralization electron density, $\rho_{e, \text { neutr }}$ is about 5 times higher than the TMCI threshold, a clear warning sign.

The electron cloud also induces coherent and incoherent tune shifts of about $\Delta Q=2 r_{e} \beta \rho / \gamma$, which, for our parameters, evaluates to $\Delta Q \approx 0.1$. This can be useful for diagnostics purposes, since measuring the tune shift allows us to monitor the electron-cloud build up and the average electron density [86]. 


\subsection{Coherent Synchrotron Radiation}

Another potentially harmful effect is coherent synchrotron radiation (CSR). At wavelengths longer than the bunch length, the bunch may radiate like a single macroparticle of charge $N_{b} e$. Since the synchrotron radiation is proportional to the charge squared, at these wavelengths the radiated power is enhanced by a factor $N_{b}$ compared with normal synchrotron radiation. This enhanced radiation is the coherent synchrotron radiation. The shorter the bunch, the larger the frequency range in which it is observed and the larger the overall effect.

The rms energy spread induced by CSR in a bend of length $L_{d}$ and radius $R$ is [88]

$$
\Delta \delta_{\mathrm{rms}}^{\mathrm{CSR}} \approx 0.2 \frac{N r_{e} L_{d}}{\gamma \rho^{2 / 3} \sigma_{z}^{4 / 3}} .
$$

The CSR is unimportant at ultra-high energies. E.g., for a bending section in the CLIC final focus $\left(N_{b}=4 \times 10^{9}, E=1.5 \mathrm{TeV}, L_{d} \approx 176 \mathrm{~m}, \theta_{d} \approx 244 \mu \mathrm{rad}, \sigma_{z} \approx 30 \mu \mathrm{m}\right)$ we have $\Delta \delta_{\mathrm{rms}}^{\mathrm{CSR}} \approx 2 \times 10^{-8}$, much smaller than the energy spread due to incoherent synchrotron radiation, $\Delta \delta_{\mathrm{rms}}^{\mathrm{SR}} \approx 10^{-5}$.

However, CSR can be important for a damping ring. CSR effects are larger for smaller bending radius and lower beam energy. In order to examine the worst case, we take numbers typical of the damping-ring lattice for a 1-TeV collider [56] rather than $3 \mathrm{TeV}$. Specifically, we assume $E=2.15$ $\mathrm{GeV}, \sigma_{z}=1.8 \mathrm{~mm}, B=1.4 \mathrm{~T}, L_{d \text {,tot }} \approx 30 \mathrm{~m}$. We find an additional induced energy spread of $\Delta \delta_{\mathrm{rms}}^{\mathrm{CSR}} \approx 2.6 \times 10^{-5}$ per turn. Further assuming $C=283 \mathrm{~m}, \alpha=2.4 \times 10^{-4}, \tau_{\delta} \approx 10.4 \mathrm{~ms}$, $\sigma_{\delta 0} \approx 8.2 \times 10^{-4}$, and considering regular synchrotron radiation as the only source of damping, we estimate the equilibrium energy spread due to CSR alone:

$$
\sigma_{\delta}^{\mathrm{CSR}} \approx \sqrt{\frac{1}{4} \tau_{E} \frac{\left(\Delta \delta_{\mathrm{rms}}^{\mathrm{CSR}}\right)^{2} c}{C}} \approx 1.3 \times 10^{-3},
$$

where $\tau_{\delta}$ is the longitudinal damping time. In this example, the energy spread induced by CSR is larger than the natural energy spread!

The low-frequency CSR will be shielded by the vacuum chamber, if the latter has a full aperture smaller than the critical aperture [89]

$$
h_{\mathrm{crit}}=\left(\pi \sigma_{z} \sqrt{R}\right)^{2 / 3}
$$

An empirical formula for the shielding efficiency obtained by fitting to a large number of computer calculations [90] is [91]

$$
\delta_{\mathrm{rms}}^{\mathrm{CSR}}(h) \approx\left(1-e^{-\frac{2 h}{h_{\mathrm{crit}}}+0.8}\right) \delta_{\mathrm{rms}}^{\mathrm{CSR}}(\infty),
$$

where $\delta_{\mathrm{rms}}^{\mathrm{CSR}}(\infty)$ denotes the energy spread induced without the shielding, Eq. (145).

As an example, with a bending radius of $R=5 \mathrm{~m}$ and a full chamber aperture $h=60 \mathrm{~mm}$ ( $a=h / 2=30 \mathrm{~mm}$ ), the CSR is shielded for bunch lengths $\sigma_{z}>2 \mathrm{~mm}$. If the full aperture can be reduced to $h=20 \mathrm{~mm}$, the CSR is shielded already for bunch lengths $\sigma_{z}>400 \mu \mathrm{m}$. However, small apertures like this may be impractical with respect to vacuum, beam lifetime, or impedance. Perhaps one may want to control the bunch length in the ring via the inductive impedance (without entering the turbulent bunch-lengthening regime) [92].

\section{RF Gun and Positron Source}

Challenges for the source design are posed by all the beam parameters required: small emittances, high charge, repetition rate, and electron polarization. 
Electron beams can be generated in a variety of ways. Accordingly a number of different devices exist which can serve as electron sources for linear colliders: thermionic guns, dc guns with laser photocathodes (used at the SLC), or rf guns. In the future, also polarized rf guns may become available.

For example, in a laser-driven rf gun, or rf photoinjector, a high-power pulsed laser illuminates a photocathode placed on the end wall of an rf cavity. The emitted electrons are accelerated immediately in the rf field. The time structure of the electron beam is controlled by the laser pulse, and the rapid acceleration minimizes the effect of the space-charge repulsion.

Several effects contribute to the normalized emittance attainable by such rf gun [93]:

(1) The thermal emittance is determined by the initial transverse momenta of the electrons at the moment of their emission,

$$
\gamma \epsilon_{x, y}^{t h}[\mathrm{~mm} \operatorname{mrad}] \approx \frac{1}{4} \sqrt{\frac{k_{B} T_{\perp}}{m_{e} c^{2}}} \sigma_{x, y}[\mathrm{~mm}],
$$

where $k_{B} T_{e} \approx 0.1 \mathrm{eV}$ represents the thermal emission temperature.

(2) An rf emittance arises from the time-dependent transverse focusing at the exit of the cavity,

$$
\gamma \epsilon_{x, y}^{\mathrm{rf}}[\mathrm{mm} \operatorname{mrad}] \approx \frac{e E_{\mathrm{rf}}}{\sqrt{8} m_{E}} \sigma_{x, y}^{2} \sigma_{z}^{2} \omega_{\mathrm{rf}}^{2},
$$

where $E_{\mathrm{rf}}$ is the peak accelerating field.

(3) The residual space-charge emittance is due to the repelling force between the equally charged beam particles [94],

$$
\gamma \epsilon_{x, y}^{\mathrm{sc}}[\mathrm{mm} \operatorname{mrad}] \approx \frac{2 N_{b} r_{e}}{7 \sigma_{x, y} W} \exp \left(-3 \sqrt{W \sigma_{y}}\right) \sqrt{\frac{\sigma_{y}}{\sigma_{z}}},
$$

where $W=e E_{\mathrm{rf}} \sin \phi_{0} /\left(2 m_{e} c^{2}\right)$ and $\phi_{0}$ the rf phase at the beam center. Since the transverse space-charge force depends on the local charge density of the bunch, it disorients in phase space the transverse slices located at different longitudinal positions along the bunch. For round beams this dilution can be almost fully inverted by properly placed solenoids [95].

Linear colliders require flat electron beams at the collision point, in order to maximize the luminosity for a certain amount of beamstrahlung. However, electron guns usually produce round beams.

\section{Case Study V: Flat RF Gun}

Conceive a scheme by which one can transform a round beam $\left(\epsilon_{x}=\epsilon_{y}\right)$ into a flat beam $\left(\epsilon_{x} \gg\right.$ $\left.\epsilon_{y}\right)$. Hint: one possibility starts with the beam from an rf gun immersed in a solenoid field, which is followed by a set of linear transformations.

Solution: A scheme for flat-round conversion was proposed by R. Brinkmann, Y. Derbenev, $K$. Floettmann in 1999 [96, 97]. We describe the idea following Ref. [98]. The basic scheme consists of two parts:

(1) the beam from a cathode which is immersed in solenoidal field develops an angular momentum at exit from solenoid;

(2) subsequently this beam is passed through a quadrupole (or skew quadrupole) channel with $90^{\circ}$ phase advance difference between the two planes, and length scale defined by the solenoid field. 
Consider electrons moving parallel to a solenoid field whose axis is oriented in z direction. Maxwell's equations imply the presence of a radial magnetic field at the exit of the solenoid. This radial field gives rise to a transverse deflection, which depends on the distance from the solenoid axis. For example, the vertical deflection at the solenoid exit reads

$$
\Delta y^{\prime}=\frac{1}{B \rho} \int B_{x} d z=\frac{1}{B \rho} \frac{x_{0}}{2} B_{z},
$$

where $B_{z}$ is the longitudinal field inside the solenoid and $x_{0}$ the horizontal offset. A similar expression holds for $\Delta x^{\prime}$. Abbreviating we write $\Delta y^{\prime}=k x_{0}, \Delta x^{\prime}=-k y_{0}$ with $k=B_{z} /(2 B \rho)$. After leaving the solenoid, the beam takes on a clock-wise rotation

$$
\left(\begin{array}{c}
x \\
x^{\prime} \\
y \\
y^{\prime}
\end{array}\right)_{0}=\left(\begin{array}{c}
x_{0} \\
-k y_{0} \\
y_{0} \\
k x_{0}
\end{array}\right)
$$

We have neglected any initial uncorrelated momenta, assuming that these are much smaller than $k x_{0}$ or $k y_{0}$. Actually, these terms are important, as they do determine the final flat-beam emittance. We will see this below.

Suppose now the quadrupole channel behind the solenoid produces an I matrix in $x$ and an additional $90^{\circ}$ phase advance in $y$ :

$$
\left(\begin{array}{c}
x \\
x^{\prime} \\
y \\
y^{\prime}
\end{array}\right)_{1}=\left(\begin{array}{cccc}
1 & 0 & 0 & 0 \\
0 & 1 & 0 & 0 \\
0 & 0 & 0 & \beta \\
0 & 0 & -1 / \beta & 0
\end{array}\right)\left(\begin{array}{c}
x_{0} \\
-k y_{0} \\
y_{0} \\
k x_{0}
\end{array}\right)=\left(\begin{array}{c}
x_{0} \\
-k y_{0} \\
k \beta x_{0} \\
-\frac{1}{\beta} y_{0}
\end{array}\right) .
$$

If we choose $\beta=1 / k$, the final phase-space vector becomes

$$
\left(\begin{array}{c}
x \\
x^{\prime} \\
y \\
y^{\prime}
\end{array}\right)_{1}=\left(\begin{array}{c}
x_{0} \\
-k y_{0} \\
x_{0} \\
-k y_{0}
\end{array}\right)
$$

This is a flat beam inclined at $45^{\circ}$. If one uses a skew quadrupole channel instead of quadrupole channel, the beam can be made flat in the vertical plane, as shown next.

The $4 x 4$ transport matrix from the end of the solenoid through the skew quadrupole channel can be written

$$
M=R^{-1} T R \quad \text { with } \quad R=\frac{1}{\sqrt{2}}\left(\begin{array}{cc}
I_{2} & I_{2} \\
-I_{2} & I_{2}
\end{array}\right),
$$

where $I_{2}$ is $2 \times 2$ identity, and the matrix $T$ represents a normal quadrupole channel:

$$
T=\left(\begin{array}{cc}
A & 0 \\
0 & B
\end{array}\right)
$$

Combining the above, we write $M$ as

$$
M=\frac{1}{2}\left(\begin{array}{cc}
A+B & A-B \\
A-B & A+B
\end{array}\right) .
$$


The initial state after the solenoid exit is

$$
X \equiv\left(\begin{array}{c}
x_{0} \\
-k y_{0}
\end{array}\right) \quad \text { and } \quad Y \equiv\left(\begin{array}{c}
y_{0} \\
k x_{0}
\end{array}\right)
$$

which we write more elegantly as

$$
Y=S X \quad \text { using } \quad S \equiv\left(\begin{array}{cc}
0 & -\frac{1}{k} \\
k & 0
\end{array}\right) .
$$

The final state is then

$$
\left(\begin{array}{l}
X \\
Y
\end{array}\right)_{1}=\frac{1}{2}\left(\begin{array}{c}
\{A+B+(A-B) S\} X \\
\{A-B+(A+B) S\} X
\end{array}\right),
$$

and the condition for a flat beam reads $Y_{1}=0$, or $I=-(A-B)^{-1}(A+B) S$.

Using the Courant-Snyder parametrization [100] $A=\exp (J \mu), B=\exp (J(\mu+\Delta))$, where $J$ denotes the matrix

$$
J=\left(\begin{array}{cc}
\alpha & \beta \\
-\gamma & -\alpha
\end{array}\right)
$$

the flat-beam condition becomes

$$
I=-\frac{\cos (\Delta / 2)}{\sin (\Delta / 2)}\left(\begin{array}{cc}
k \beta & \alpha / k \\
-k \alpha & \gamma / k
\end{array}\right)
$$

This is fulfilled for $\Delta=-\pi / 2, \alpha=0$ and $\beta=1 / k$.

Finally, adding a random component to the slope to the initial vector $\left(x, x^{\prime}, y, y^{\prime}\right)_{0}=$ $\left(x_{0},-k y_{0}+x_{0}^{\prime}, y_{0}, k x_{0}+y_{0}^{\prime}\right)$ one can apply the same transformation $M$ and, assuming that the initial beam is round with $\sigma_{x 0}=\sigma_{y 0}$ and $\sigma_{x 0}^{\prime}=\sigma_{y 0}^{\prime}$, one finds [96]

$$
\epsilon_{y, 1}=\frac{1}{2} \frac{\sigma_{x, y}^{\prime 2}}{k}
$$

and

$$
\epsilon_{x, 1} / \epsilon_{y, 1}=1+4 k^{2} \frac{\sigma_{x, y}^{2}}{\sigma_{x, y}^{\prime}{ }^{2}} .
$$

The larger $k$, i.e., the stronger the solenoid field, the flatter the beam becomes.

First experimental tests of a flat beam electron source at Fermilab have demonstrated the viability of this scheme [99].

The conventional approach to produce positron beams is to hit a high- $\mathrm{Z}$ target with a several-GeV $\mathrm{e}^{-}$beam. An electro-magnetic shower of bremsstrahlung and pair creation develops, in the course of which a large number of positrons are produced. In order to get sufficiently many positrons, a thick high- $\mathrm{Z}$ material is chosen as a target. This method was used at the SLC and it is the preferred option for NLC.

The TESLA project considers an alternative approach of generating the positron beam. Here, a high-energy electron beam passing through a wiggler emits hard synchrotron-radiation photons which impact on a thin target downstream and produce positrons via pair creation [8, 101, 102]. A schematic of the TESLA positron source is shown in Fig. 16. In this design, a thin low-Z target is sufficient to produce the desired number of positrons. Main advantages are, firstly, the lower heat capacity $C_{p}$, and hence, the smaller target temperature rise, and, secondly, the reduced scattering. 


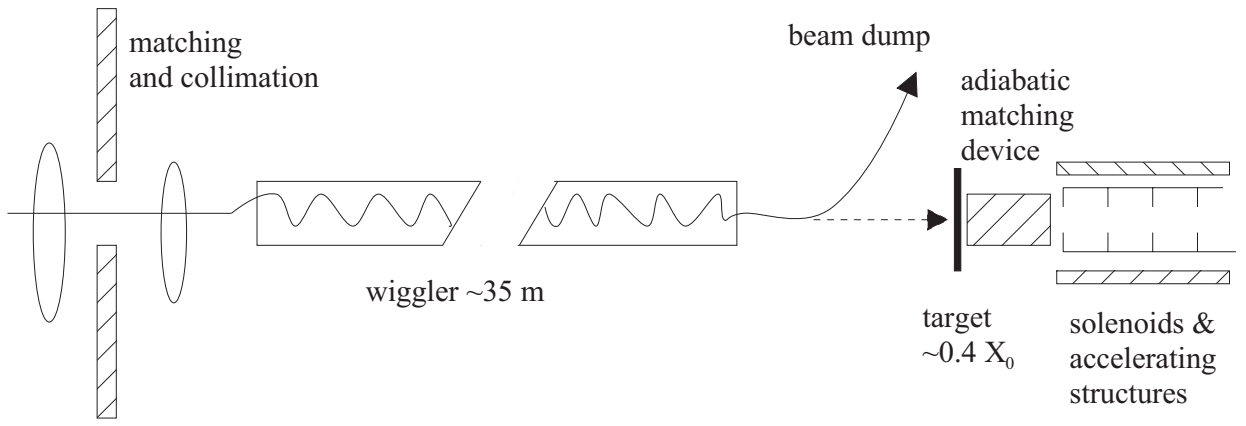

Figure 16: Schematic of TESLA positron source, consisting of a wiggler, a thin low-Z target, and a capture section with adiabatic matching [8].

The latter implies a smaller positron divergence and a better capture efficiency. The TESLA source is even capable of producing polarized positrons if the wiggler is replaced by a helical undulator.

The positron capture section behind the photon target consists of acceleration units embedded in a strong solenoid field. The acceptance of the solenoid channel is well suited for a large spot size and small angles, whereas the positrons emerge from the target with a small spot size and large angles. A tapered solenoid with adiabatically increasing field strength [103] provides the optical matching between the two regions.

\section{Acknowledgements}

I am grateful to R. Assmann, M. Minty, F. Ruggiero, F. Schmidt, D. Schulte, and J. Urakawa for various helpful discussions and generous contributions. I thank F. Ruggiero, M.-P. Zorzano, H. Burkhardt, and E. Keil, for a careful reading of the manuscript and valuable comments. I also owe many thanks to the students who joined this tutorial. Without their active participation it would have been much less exciting. Their questions greatly inspired the written document. Finally, I thank the organizers of the Joint Accelerator School, in particular, E. Wilson, S.Y. Lee, J. Miles, J. Murphy, and E. Perevedentsev, for their constant encouragement and for providing the possibility for this tutorial, and my co-teacher P. Logachev for his cheerful collaboration. 


\section{References}

[1] M. Tigner, “A Possible Apparatus for Electron-Clashing Experiments," Nuovo Cimento p. 3944 (1965).

[2] U. Amaldi, "A Possible Scheme to Obtain $\mathrm{e}^{+} \mathrm{e}^{-}$Collisions at Energies of Hundreds of GeV," Physics Letters 61B no. 3 (1976).

[3] R. Erickson (ed.) et al., "SLAC Linear Collider Design Handbook," Stanford Linear Accelerator Center (1984).

[4] J. Seeman, K.L. Bane T. Himel, W.L. Spence, C. Adolphsen, "Observation and Control of Emittance Growth in the SLC Linac," 14th Int. Conference on High Energy Accelerators, Tsukuba, Japan, Part. Accel. 30, 97 (1990).

[5] R. W. Assmann, "Beam dynamics in SLC," 17th IEEE Particle Accelerator Conference (PAC 97), Vancouver, Canada, and SLAC-PUB-7576 (1997).

[6] R. W. Assmann et al., 'Accelerator physics highlights in the 1997/98 SLC run," 1st Asian Particle Accelerator Conference (APAC 98), Tsukuba, Japan, and SLAC-PUB-7782 (1998).

[7] P. Raimondi et al., "Recent luminosity improvements at the SLC," 17th International Conference on High-Energy Accelerators (HEACC 98), Dubna, Russia, and SLAC-PUB-7955 (1998).

[8] R. Brinkmann et al. (eds.), "Conceptual Design of a $500 \mathrm{GeV} \mathrm{e}^{+} \mathrm{e}^{-}$Linear Collider with Integrated X-ray Laser Facility,” DESY 1997-048, ECFA 1997-182 (1997).

[9] C. Adolphsen at el., "NLC Zeroth Order Design Report for the Next Linear Collider," SLAC Report 474 (1996).

[10] J.P. Delahaye and I. Wilson, “CLIC a multi-TeV e+e- Linear Collider,” CERN/PS 99-062 (LP)

[11] The CLIC Study Group, G. Guignard (ed.), "General Description of a 3 TeV Linear Collider based on the CLIC Technology," CERN Report 2000-008 (2000).

[12] A. Chao, Physics of Collective Beam Instabilities in High Energy Accelerators, J. Wiley (1993).

[13] H. Padamsee, J. Knobloch, T. Hays, RF Superconductivity for Accelerators, J. Wiley (1998).

[14] E. Jensen, private communication (2000).

[15] M. Chodorow, et al., Rev. Sci. Instrm. 26 p. 134 (1955).

[16] A.E. Vlieks, et al., "Breakdown phenomena in High-Power Klystrons," SLAC-PUB-4546 (1988).

[17] D. Whittum, H. Henke and P. Chou, "High-Gradient Cavity Beat Wave Accelerator at W Band," PAC 97 and SLAC-PUB-7805 (1998).

[18] A similar formula was presented by R. Brinkmann around 1992.

[19] K. Yokoya and P. Chen, "Beam-beam phenomena in linear colliders," Lecture at 1990 USCERN School on Particle Accelerators, Hilton Head Island, South Carolina (1990).

[20] M. Sands, “The Physics of Electron Storage Rings,” SLAC Report 121 (1970).

[21] P. Chen, "Differential Luminosity under Multi-Photon Beamstrahlung," Physical Review D46, 1186 (1992).

[22] V.E. Balakin and N.A. Solyak, Proc. XIIIth Int. Conf. on High Energy Accelerators, Novosibirsk (1986). 
[23] J.B. Rosenzweig, B. Autin, P. Chen, "Instability of Compensated Beam-Beam Collisions," 1989 Lake Arrowhead Workshop on Advanced Accelerator Concepts (1989).

[24] D. H. Whittum, A. M. Sessler, J. J. Stewart and S. S. Yu, "Plasma Suppression Of Beamstrahlung," Part. Accel. 34, 89 (1990).

[25] I. F. Ginzburg, G. L. Kotkin, V. G. Serbo and V. I. Telnov, "Colliding Gamma e and Gamma Gamma Beams Based on the Single Pass Accelerators (of VLEPP Type)," Nucl. Instrum. Meth. 205, 47 (1983).

[26] V. Telnov, "Principles of photon colliders," Nucl. Instrum. Meth. A355, 3 (1995).

[27] K. L. Brown, "Basic Optics of the SLC Final Focus System," SLAC-PUB-4811; presented at Workshop on Physics of Linear Colliders, Capri, Italy, Jun 13-17, 1988 and at Int. Workshop on the Next Generation of Linear Colliders, Stanford, CA, Nov 28 - Dec 9, 1988.

[28] K.L. Brown, "A First and Second Order Matrix Theory for the Design of Beam Transport Systems and Charged Particle Spectrometers," Adv. Part. Phys. 1, 71 (1968).

[29] P. Emma, D. McCormick, M.C. Ross, "Beam Dispersion Measurements with Wire Scanners in the SLC Final Focus System," PAC93, Washington, and SLAC-PUB-6208 (1993).

[30] F. Zimmermann, "New Final-Focus Concepts at $5 \mathrm{TeV}$ and Beyond," 8th Workshop on Advanced Acceleration Concepts, Baltimore, Maryland, and SLAC-PUB-7883 (1998).

[31] P. Raimondi and A. Seryi, "A Novel Final-Focus Design for High-Energy Linear Colliders," EPAC 2000, Vienna (2000).

[32] S. Fartoukh and J.B. Jeanneret, "Using Microwave Quadrupoles to Shorten the CLIC Beam Delivery Section," EPAC 2000, Vienna (2000).

[33] H. Burkhardt, "Background in Future $e^{+} e^{-}$Linear Colliders," CERN-SL-057-AP and CLIC Note 416 (1999).

[34] R. Brinkmann, talk at International Linear Collider Workshop LC99, Frascati (1999).

[35] F. J. Decker et al., "Design and wakefield performance of the new SLC collimators," LINAC96, Geneva, and SLAC-PUB-7261 (1996).

[36] R. Brinkmann, et al., TESLA 95-25 (1995).

[37] R. Assmann, et al., "Design Status of the CLIC 3-TeV Beam Delivery System and Damping Rings," presented at EPAC 2000, Vienna (2000).

[38] J. Frisch, E. Doyle, K. Skarpaas VIII, “Advanced Collimator Systems for the NLC,” Proc. of Linac 2000 Monterey (2000).

[39] Ralph Assmann and Alex Chao, "Dispersion in the Presence of Strong Transverse Wakefields," PAC97, Vancouver, and SLAC-PUB-7581 (1997).

[40] F. Ruggiero and A. Zholents, "Resonant Correction of Residual Dispersion in LEP," CERN SL MD Note 26 (1992).

[41] D. Schulte, "Emittance Preservation in the Main Linac of CLIC," EPAC98, Stockholm, and CERN-PS-98-018-LP (1998).

[42] T. Raubenheimer, "The Generation and Acceleration of Low Emittance Flat Beams for Future Linear Colliders,” Ph.D. thesis, Stanford University, and SLAC Report 387 (1991).

[43] R. Assmann, T. Chen, F.J. Decker, M. Minty, P. Raimondi, T.O. Raubenheimer, R. Siemann, "Simultaneous Trajectory and Dispersion Correction in the SLC Linac," unpublished. 
[44] V. Balakin, A. Novokhatsky, V. Smirnov, 12th Int. Conf. on High Energy Accelerators, Fermilab (1983).

[45] W. Schnell, “Microwave Quadrupoles for Linear Colliders,” CLIC Note 34 (1987).

[46] T. Raubenheimer and R. D. Ruth, "A Dispersion free trajectory correction technique for linear colliders," Nucl. Instrum. Meth. A302, 191 (1991).

[47] V. Ziemann, “Corrector Ironing,” SLAC-CN-393 (1992)

[48] P. Raimondi implemented the SVD algorithm for dispersion-free steering at the SLC.

[49] T. Raubenheimer and R. D. Ruth, "A New Method of Correcting the Trajectory in Linacs," IEEE PAC San Francisco (1991).

[50] R. Assmann et al., "The computer program LIAR for the simulation and modeling of high performance linacs," 17th IEEE PAC 97, Vancouver, Canada, and SLAC-PUB-7577 (1997).

[51] G. Guignard and J. Hagel, "Mustafa environment description and users' guide with applications to CLIC," CERN-SL-98-002-AP and CLIC-Note-349 (1998).

[52] D. Schulte, "PLACET: A program to simulate drive beams," CERN-PS-2000-028-AE (2000).

[53] R.W. Assmann, "Beam dynamics in SLC," IEEE PAC 97, Vancouver, Canada, and SLACPUB-7576 (1997).

[54] J. Jowett, private communication (2000).

[55] L. Teng, "Minimizing the Emittance in Designing the Lattice of an Electron Storage Ring," FNAL TM-1269 (1985).

[56] J.P. Potier and L. Rivkin, “A Low Emittance Lattice for the CLIC Damping Ring,” IEEE PAC 1997 Vancouver (1997).

[57] J. Le Duff, “Single And Multiple Touschek Effects," CERN Accelerator Schools, West Berlin and Rhodes, CERN-95-06 (1995).

[58] M. S. Zisman, S. Chattopadhyay and J. J. Bisognano, “Zap User’s Manual,” LBL-21270 (1986).

[59] T. O. Raubenheimer, "The core emittance with intrabeam scattering in e+/e- rings," Part. Accel. 45, 111 (1994).

[60] F. Hinode et al., “ATF Design and Study Report,” KEK Internal 95-4 (1995).

[61] A. Hofmann and F. Meot, "Optical Resolution Of Beam Cross-Section Measurements By Means Of Synchrotron Radiation,” Nucl. Instrum. Meth. 203, 483 (1982).

[62] O. Chubar, “Novel Applications of Optical Diagnostics,” EPAC 2000 Vienna (2000).

[63] T. Weitkamp et al., "Electron Beam Profile Measurements with Refractive X-Ray Lenses," EPAC 2000 Vienna (2000).

[64] P. Elleaume, C. Fortgang, C. Penel, E. Tarazona, "Measuring Beam Sizes and Ultra-Small Electron Emittances Using an X-Ray Pinhole Camera,” J. Synchrotron Rad. 2, 209 (1995).

[65] T. Mitsuhashi, "Beam Profile and Size Measurement by SR Interferometers," Joint US-CERNRussia-Japan School on Particle Accelerators: Beam Measurement, Montreux (1998).

[66] T. Mitsuhashi and T. Naito, "Measurement of Beam Size at ATF Damping Ring with the SR Interferometer," EPAC Stockholm and ATF Report 98-17 (1998).

[67] A. Piwinski, "Intrabeam Scattering," Ninth International Conference on High Energy Accelerators, Stanford 1974, Springfield (1975); see also CERN Accelerator School, Oxford 1985, (1985). 
[68] J. D. Bjorken and S. K. Mtingwa, “Intrabeam Scattering,” Part. Accel. 13, 115 (1983).

[69] C. Bernadini et al., Phys. Rev. Letters 10, p. 407 (1963).

[70] R.P. Walker, "Calculation of the Touschek Lifetime in Electron Storage Rings," PAC 1987.

[71] H. Sakai, private communication (2000).

[72] J. Urakawa, M. Ross, and T. Raubenheimer, private communications (1998).

[73] L. Arnaudon et al., "Effects of Terrestrial Tides on the LEP Beam Energy," Nucl. Instrum. Meth. A357, 249 (1995).

[74] D. Boussard, CERN Lab II/RF/Int 75-2 (1975).

[75] E. Keil and W. Schnell, CERN Report TH-RF/69-48 (1969); V.K. Neil and A.M. Sessler, Rev. Sc. Instr. 36, 429 (1965).

[76] T. O. Raubenheimer and F. Zimmermann, "A Fast beam - ion instability in linear accelerators and storage rings," Phys. Rev. E52, 5487 (1995).

[77] G. V. Stupakov, T. O. Raubenheimer and F. Zimmermann, "Fast beam ion instability. 2. Effect of ion decoherence," Phys. Rev. E52, 5499 (1995).

[78] J. Byrd, A. Chao, S. Heifets, M. Minty, T. O. Raubenheimer, J. Seeman, G. Stupakov, J. Thomson, and F. Zimmermann, "First Observations of a "Fast Beam-Ion Instability"," Physical Review Letters 79, 1, 79 (1997).

[79] J. Y. Huang, M. Kwon, T.-Y. Lee, I.S. Ko, Y. H. Chin, H. Fukuma "Direct Observation of the Fast Beam-Ion Instability,” Phys. Rev. Letters 81, 4388 (1998).

[80] R. Nagaoka, J.L. Revol, J. Jacob, “Observation, Analysis and Cure of Transverse Multibunch Instabilities at the ESRF," EPAC 2000 Vienna (2000).

[81] G.V. Stupakov, “A Fast Beam Ion Instability,” Proc. of Int. Workshop on Collective Effects and Impedance for B factories (CEIBA95), KEK Proceedings 96-6, p. 243 (1996).

[82] K. Ohmi, “Beam Photoelectron Interactions in Positron Storage Rings," Phys. Rev. Lett. 75, 1526 (1995).

[83] M. Izawa, Y. Sato, and T. Toyomasu, "The vertical instability in a positron bunched beam," Phys. Rev. Lett. 74, 25, p. 5044 (1995).

[84] Z.Y. Guo et. al., "Study of the beam-photoelectron instability in BEPC," 1st APAC Tsukuba (1998).

[85] K. Ohmi and F. Zimmermann, "Head-Tail Instability caused by Electron Cloud in Positron Storage Rings," submitted to Phys. Rev. Letters, and CERN-SL-2000-015 AP (2000).

[86] H. Fukuma, et al., "Observation of Vertical Beam Blow-Up in KEK Low Energy Ring," presented at EPAC 2000 Vienna (2000).

[87] Proceedings of Chamonix X, in particular contributions by W. Hoefle, M. Jimenez, and G. Arduini, CERN-SL-2000-007 (DI) (2000).

[88] Ya.S. Derbenev, J. Rossbach, E.L. Saldin, and V.D. Shiltsev, "Microbunch Radiative Head-Tail Interaction,” DESY TESLA-FEL 95-09 (1995).

[89] R.L Warnock, "Shielded Coherent Synchrotron Radiation and Its Possible Effect in the Next Linear Collider,” Proc. IEEE PAC 1991 San Francisco and SLAC-PUB-5523 (1991).

[90] R.L. Warnock and P. Emma, unpublished.

[91] P. Emma, private communication (1996). 
[92] F. Ruggiero, private communication (2000).

[93] K.-J. Kim, "RF and Space-Charge Effects in Laser-Driven RF Electron Guns," Nucl. Instr. Methods A275, 201 (1989).

[94] J.B. Rosenzweig, E. Colby, G. Jackson, T. Nicol, "Design of a High Duty Cycle, Asymmetric Emittance RF Photoinjector for Linear Collider Applications,", Proc. IEEE PAC 1993 Washington D.C. (1993).

[95] M.E. Jones and B.E. Carlsten, "Space-Charge Induced Emittance Growth in the Transport of High-Brightness Electron Beams,” IEEE PAC Washington (1987).

[96] R. Brinkmann, Ya. Derbenev, K. Flöttmann, “A Flat Beam Electron Source for Linear Colliders," TESLA 99-09 (1999).

[97] R. Brinkmann, Y. Derbenev, K. Flöttmann, “A Low Emittance, Flat-Beam Electron Source for Linear Colliders," Proc. EPAC 2000, Vienna (2000).

[98] D. Edwards, "Notes on the Production of Flat Beams," unpublished, dated January 17, 2000 (2000).

[99] D. Edwards, H. Edwards, N. Holtkamp, S. Nagaitsev, J. Santucci, R. Brinkmann, K. Desler, K. Floettmann, I. Bohnet, M. Ferrario, "The Flat Beam Experiment at the FNAL Photoinjector," presented at LINAC 2000, Monterey (2000).

[100] E.D. Courant and H.S. Snyder, “Theory of the Alternating Gradient Synchrotron," Annals Phys. 281, 360 (1958).

[101] V.E. Balakin and A.A. Mikhailichenko, "The Conversion System for Obtaining High Polarized Electrons and Positrons," Preprint INP 79-85 (1979).

[102] K. Flöttmann, "Investigations Toward the Development of Polarized and Unpolarized High Intensity Positron Sources for Linear Colliders,” DESY-93-161 (1993).

[103] R. Helm, "Adiabatic Approximation for Dynamics of a Particle in the Field of a Tapered Solenoid," SLAC-4 (1962). 